\title{
A Mechatronic Analysis of the Classical Position-Force controller based on Bounded Environment Passivity
}

\author{
B. Willaert, B. Corteville, D. Reynaerts, H. Van Brussel \\ and E.B. Vander Poorten
Department Mechanical Engineering, K.U.Leuven
Celestijnenlaan 300, 3001 Heverlee, Belgium

email: bert.willaert@mech.kuleuven.ac.be

June 15, 2010

\begin{abstract}
Bounded Environment Passivity, presented in this paper, allows one to design teleoperation systems that behave passive provided that the environment with which interaction takes place, belongs to an a-priori defined range of environments. The use of such a-priori knowledge on the environment reduces conservativeness with respect to classical design approaches. An additional advantage lies in its capability to get a clearer insight on which type of environments are problematic for the specific controller under investigation.

On the basis of a case study, i.e. the well-known Position-Force controller, this paper describes and compares different passivity-based methods. First, the traditional methods of two-port passivity and absolute stability are applied. The restrictions of these methods to come up with useful design rules are explicitly demonstrated. Then, the Bounded Environment Passivity conditions of the Position-Force controller are derived. These conditions describe the relation between the specific controller implementation, the teleoperator dynamics and the environment characteristics. Additionally, the effects of structural resonance frequencies and low-pass filters, often present in realistic teleoperator setups, are described. This analysis reveals fundamental mechatronic rules of thumb for the design of a teleoperator system with a Position-Force control architecture. The theoretical results are verified experimentally on a 1-d.o.f.-teleoperation system.

Keywords: Teleoperation control - Passivity - Stability - Mechatronics.
\end{abstract}




\section{Introduction}

A teleoperation system allows a human operator to manipulate a remote environment through a master and slave robotic device. When this teleoperation system contains a bilateral controller, haptic feedback is provided to the operator, which enables him/her to feel the remote environment. Currently, teleoperation systems are frequently used for surgical procedures Hockstein et al. 2007. Despite the fact that several studies have shown that haptic feedback would increase the precision of telesurgery and lower the interaction forces with the tissue Deml et al., 2005, Wagner et al., 2002, Tholey et al., 2005, this feature is not present in current commercial systems. The realization of reliable haptic feedback remains a real challenge including both hardware development and control design.

A prerequisite for successful haptic feedback, especially in telesurgery, is guaranteed stability of the teleoperation system. In literature, several approaches to stability have been proposed. One can calculate the closed-loop stability of a teleoperation system for a fixed operator and environment, but the robustness of this approach is very limited. This is because the behaviour of the human operator is known to be highly variable Hannaford and Anderson, 1988 , Hogan, 1989 and, depending on the application, the exact properties of the environment are not or only vaguely known a priori. A more robust approach to study stability of a teleoperation system is to use passivity theory Desoer and Vidyasagar, 1975, i.e. to analyze stability from an energy exchange point of view rather than from a signal control point of view. Two-port passivity Raisbeck, 1954, Raju et al., 1988, Anderson and Spong, 1988, Colgate 1993, Yokokohji and Yoshikawa, 1994] and absolute stability Llewellyn, 1952 Adams and Hannaford, 1999, Hashtrudi-Zaad and Salcudean, 2001, Cho and Park, 2005, Colgate, 1993, Vander Poorten et al. |2006 are two sufficient conditions for stable interaction with an arbitrary passive operator and environment. However, these two classical methods to study the stability of a teleoperation system have some drawbacks: (i) For some control structures one can prove that the teleoperation system is never two-port passive or absolutely stable for arbitrary values of the control parameters. For these control structures, these classical methods do not result in useful conditions on the parameters of the system. (ii) Even if these classical methods result in useful conditions, for some applications these conditions can be (too) conservative, as stability is guaranteed for the whole range of passive operators and environments. Besides the above mentioned problems of availability and conservativeness of the resulting conditions, (iii) the classical methods do not provide insight into which type or range of operators or environments is problematic.

Recently, Willaert et al. 2009] proposed a new method, also based on passivity theory, to tackle these problems. The idea of this method, referred to as Bounded Environment Passivity, is to analyze the passivity of the one-port network $Y_{M S}$, shown in Fig. 1. consisting of both the teleoperation system and the environment. The Bounded Environment Passivity method, checks the passivity for a pure mass environment and a pure stiffness environment separately. Furthermore, the method allows the incorporation of bounds on the magnitude of this pure mass and pure stiffness. This method results in practical conditions by calculating the positive-realness of the one-port network $Y_{M S}$ for a pure mass 
$M_{e}$ and a pure stiffness $K_{e}$ both with a bounded magnitude ${ }^{1}$

$$
\begin{gathered}
Y_{M S\left(M_{e}\right)} \text { BIBO-stable and } \Re\left(Y_{M S\left(M_{e}\right)}\right) \geq 0 \text { with } M_{e} \in\left[0, M_{e}^{\max }\right], \\
Y_{M S\left(K_{e}\right)} \text { BIBO-stable and } \Re\left(Y_{M S\left(K_{e}\right)}\right) \geq 0 \text { with } K_{e} \in\left[0, K_{e}^{\max }\right] .
\end{gathered}
$$

Note that it is shown in this paper that guaranteeing the positive-realness of $Y_{M S\left(M_{e}\right)}$ for all $M_{e} \in[0, \infty]$ and of $Y_{M S\left(K_{e}\right)}$ for all $K_{e} \in[0, \infty]$ results in the same conditions as given by absolute stability. This should be no surprise as a pure stiffness and a pure mass are from passivity point of view the worst case environments Colgate and Hogan, 1988.

Complementary to the Bounded Environment Passivity method, Bounded Operator Passivity could be studied, i.e. calculating the passivity of the one-port network $Y_{S M}$ that consists of both the operator and the teleoperation system. In this paper, only the Bounded Environment Passivity method is applied, as this method is expected to provide the most useful insights for the PositionForce controller investigated here. Both the Bounded Environment Passivity and Bounded Operator Passivity method run parallel to efforts by Adams and Hannaford 2002, Cho and Park 2005] and Vander Poorten 2007] who use passive shunt impedances to restrict the dynamic range of the environment and/or operator that is taken into account. A more recent method presented by Haddadi and Hashtrudi-Zaad 2008 allows to incorporate bounds directly on the environment and/or operator by using the scattering domain. In comparison to the presented control analyses based on the aforementioned methods, the analysis presented in this paper provides analytic design and tuning guidelines for the Position-Force controller to a level of detail not yet presented in literature before, as far as the authors are aware of. Obtaining such analytic guidelines can be simplified by only looking at the so-called worst case environments (or operators) separately. This restricts the number of parameters in the admittance $Y_{M S}$ (or $Y_{S M}$ ) and thus facilitates analytic analyses.

\footnotetext{
${ }^{1}$ For some controllers, in an application where there is guaranteed contact with the environment, it can be interesting to also include lower limits $M_{e}^{\text {min }}$ and $K_{e}^{\text {min }}$ for the pure mass and the pure stiffness. In case of the Position-Force controller investigated here, however, including such lower limits does not affect the resulting passivity conditions.
}

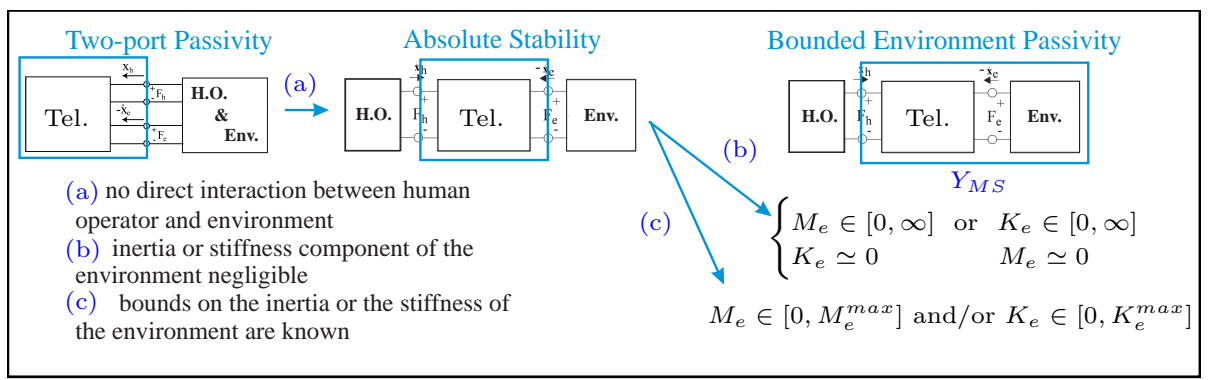

Figure 1: A task-based passivity analysis: depending on the available knowledge on the human operator and the environment, i.e. (a), (b) or (c), different passivity-based methods can be used. 
This paper describes how the use of the classical methods and the Bounded Environment Passivity method allows for a task-based, mechatronic analysis resulting in design and tuning guidelines. The analysis is task-based as, depicted in Fig. 1. the passivity of $Y_{M S}$ can be guaranteed for the whole range of passive environments up to a very specific range of environments, depending on the available knowledge on the environment. For example, if the inertial properties of the environment can be assumed negligible, it suffices to guarantee passivity of $Y_{M S}$ only for pure stiffnesses and not for pure masses. Furthermore, for many surgical procedures, it is relatively easy to delineate a range of stiffnesses $\left[0, K_{e}^{\max }\right]$ with which the surgeon interacts. Based on the raw data acquired by e.g. Walraevens et al. 2008 (cardiovascular tissue) and Rosen et al. 2008 (abdominal organs), one can estimate that the maximum stiffness is in the order of $1500 \mathrm{~N} / \mathrm{m}$ when interacting with soft tissue. When contacting bone, the stiffness can be up to $8000 \mathrm{~N} / \mathrm{m}$ Bankman et al., 1990]. If none of these assumptions about the environment are applicable, one can fall back on the classical methods. In that case, if direct interaction between the operator and the environment is impossible, it is generally accepted to use absolute stability instead of two-port passivity, as two-port passivity is a more conservative property neglecting this knowledge. Note that, even in the case that no knowledge on the environment is available, the Bounded Environment Passivity method has the advantage of giving a clearer insight into which type of environments are problematic for a specific controller.

In this paper, the Position-Force controller is chosen as a case study for the task-based mechatronic analysis mentioned above. This controller commands the slave robot to follow the position of the master robot and reflects the interaction force, measured at the slave side, to the master side. It has been proposed as a controller for telesurgery by, among others, Cavusoglu et al. [2002], De Gersem et al. 2005 and Tavakoli et al. 2007. Other researchers, such as Misra and Okamura 2006, Willaert et al. [2010b and Son and Lee 2008] compare alternative controllers to the Position-Force controller.

The success of the Position-Force controller lies in its simplicity. It is relatively easy to implement and shows a reasonably good performance. Its main limitation, however, is its stability properties. Especially for stiff environments stability problems are being reported Lawrence, 1993, Aliaga et al., 2004, Hannaford and Anderson, 1988, Daniel and McAree, 1998. As its stability problems are widely known, the Position-Force controller is an interesting controller for an in-depth stability/passivity analysis.

Researchers have spent a considerable amount of effort to study the stability properties of the Position-Force controller. Several researchers have studied closed loop stability of the Position-Force controller encompassing operator and environment. Hannaford 1989b analyzes the effect of the impedance of the operator, the impedance of the environment and the force scaling factor on the loop gain of the closed loop system. Daniel and McAree 1998 analyse the pole-locations of this closed-loop system. Although they claim to investigate the fundamental limits of the Position-Force controller, it does not present analytic conditions including the effect of all parameters of the system. Fite et al. 2004 apply loop-shaping techniques in order to improve the stability of 
the closed loop system. Other researchers studied the absolute stability properties of the Position-Force controller based on Llewellyn's absolute stability criteria Llewellyn, 1952, Adams and Hannaford, 1999]. Tavakoli et al. 2007. proved analytically that a specific implementation of the Position-Force controller cannot be absolutely stable, while Hashtrudi-Zaad and Salcudean 2001] investigated among other controllers the Position-Force controller based on a numerical evaluation of Llewellyn's absolute stability criteria. However, none of the aforementioned works described analytic closed-form passivity conditions, which allow, furthermore, the incorporation of bounds on the environment.

The teleoperation system is described in detail in section 2. The Two-port Passivity and the Absolute Stability properties of the Position-Force controller are studied in section 3 and section 4 respectively. The Bounded Environment Passivity method is described and applied in section 5 while section 6 studies the effect of a low-pass filter in the loop and the effect of a structural resonance frequency of the slave robot. In section 7 , the transparency aspects of the Position-Force controller are described. Experimental verification of the proposed method is given in section 8 . The paper ends with the conclusions and the description of future work in section 9 .

\section{The Position-Force controller}

The analysis in this paper is mainly based on 1-d.o.f rigid-body models for master and slave, obeying the following equations of motion:

$$
\begin{gathered}
F_{h}+\tau_{m}=Z_{m} v_{m}, \\
\tau_{s}-F_{e}=Z_{s} v_{s},
\end{gathered}
$$

with $Z_{m}$ and $Z_{s}$ representing the rigid body impedances of the master and the slave robot:

$$
Z_{m}=M_{m} s+B_{m}, \quad Z_{s}=M_{s} s+B_{s} .
$$

Remark that for a rigid body model the velocities $v_{m}$ and $v_{s}$ (the velocities of the motors) are equal to $v_{h}$ and $v_{e}$ respectively (the velocities of the end-effectors). The basic configuration of the Position-Force controller is illustrated in Fig. 2 The control input forces to the motors of the master and the slave are:

$$
\begin{gathered}
\tau_{m}=-\lambda F_{e} \\
\tau_{s}=C_{s}\left(\mu x_{m}-x_{s}\right),
\end{gathered}
$$

with $\mu$ and $\lambda$ the position and force scaling factor and $x_{m}$ and $x_{s}$ the positions of the motors. Note that here a low-impedance-type ${ }^{2}$ master is assumed and pure open-loop force control is used. The compensator $C_{s}(s)$ of the closed-loop position controller is typically a PD-controller ${ }^{3} K_{v} s+K_{p}$. This configuration has been studied by Lawrence [1993], Hashtrudi-Zaad and Salcudean 2001, Fite et al. 2001 and Tavakoli et al. 2007. An alternative controller, used by

\footnotetext{
${ }^{2}$ A low-impedance-type device is characterized by low inertia and low friction/damping. The PHANToM haptic device is a good example of a low-impedance-type device.

${ }^{3}$ Note that there are typically two conventions for a position controller, i.e. $\mathrm{P} / \mathrm{PD}$ on position or I/PI on velocity.
} 


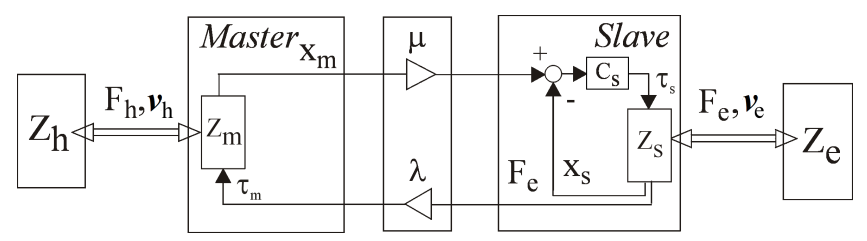

Figure 2: Representation of the classic Position-Force controller (PD-F scheme)

Table 1: Parameters of the teleoperation system

\begin{tabular}{|ll|ll|}
\hline \multicolumn{2}{|l|}{ Model } & \multicolumn{2}{|c|}{ Controller } \\
\hline \hline$M_{m}:$ & $0.64 \mathrm{~kg}$ & $K_{v}:$ & $80 \mathrm{Ns} / \mathrm{m}$ \\
$B_{m}:$ & $3.4 \mathrm{Ns} / \mathrm{m}$ & $K_{p}:$ & $4000 \mathrm{~N} / \mathrm{m}$ \\
$M_{s}:$ & $0.61 \mathrm{~kg}$ & $\mu:$ & 1 \\
$B_{s}:$ & $11 \mathrm{Ns} / \mathrm{m}$ & $\lambda:$ & 1 \\
\hline
\end{tabular}

Yokokohji and Yoshikawa 1994 and Daniel and McAree 1998, differs in the fact that it does not contain a master velocity feedforward term. The control input for the slave is then given by:

$$
\tau_{s}=K_{p}\left(\mu x_{m}-x_{s}\right)-K_{v} v_{s}
$$

As far as the authors are aware of, a concise comparison of the difference between both configurations has not yet been described. In this paper both configurations will be covered. For clarity the approach based on (77) will be referred to as the PD-F scheme, whereas the one based on (8) will be called the $\mathbf{P}-\mathbf{F}$ scheme.

All the numerical representations of analytic conditions (section 4 and 5), all the numerical calculations (section 6) and all the experiments (section 8) reported in this paper are based and done, respectively, on a 1-d.o.f. experimental masterslave setup shown in Fig 13 . This setup consists of two current-driven voice coil motors from hard disk drives. On both devices, one-dimensional force sensors are mounted, measuring the interaction forces between slave and environment at one side, and between the human operator and the master at the other side (noise-level: $0.05 \mathrm{~N}$, full range: $\pm 50 \mathrm{~N}$ ), while linear encoders offer accurate position measurements (resolution: $1 \mu \mathrm{m}$, full range: $50 \mathrm{~mm}$ ). The velocity signals are obtained through differentiation of the position signal, and are subsequently filtered with a $2^{\text {nd }}$ order low-pass filter with a bandwidth of $80 \mathrm{~Hz}$. The structural resonance frequencies of both the master and the slave device are above $100 \mathrm{~Hz}$. Table 1 gives an overview of the used parameters for the rigid-body models and the controllers. These parameters, based on the linear model identification of the master and slave and on the implementation of the controllers on this setup, serve as a starting point for the analysis in this paper. The controllers are implemented on a dSpace board, in a real-time control loop with a frequency of $1 \mathrm{kHz}$. Note that both the master and the slave suffer from Coulomb friction with a magnitude varying between 0.5 and $1 \mathrm{~N}$. Because this analysis is based on linear systems, the Coulomb friction is not explicitly included in the model for the master and slave. However, it is implicitly included. A frequency-based identification was performed and resulted in the damping value of Table 1 following a linear model fit on the measured frequency response. The obtained value thus partially represents the linearized Coulomb 
friction and partially the viscous friction.

\section{Two-port Passivity Analysis}

Passivity has turned out to be an interesting property for the study of humanrobot interaction. As described by Desoer and Vidyasagar [1975, it is an inputoutput property of dynamical systems dealing with the exchange of energy between interconnected systems . Passivity is well-known to be a sufficient condition for a system to guarantee stable interaction of the system with any passive environment and/or human operator. In the case of teleoperation, the robotic system contains both a master and a slave robot, which interact with the human operator and the external environment. Fig. 3 shows a network model of such a teleoperation system. To evaluate the 2-port passivity of a teleoperation system, one can look at the positive-realness of the immittance matrix ${ }^{4}$ Raju et al. 1988, Colgate, 1993], the bounded-realness of the scattering matrix Anderson and Spong, 1988, Colgate, 1993, Yokokohji and Yoshikawa, 1994, or the Raisbeck passivity criterion Raisbeck, 1954]. The latter is used here and can be expressed in function of the elements $h_{i j}$ of the hybrid matrix $\mathbf{H}$ of the teleoperation system which describes the relationship between the efforts and flows as follows:

$$
\left[\begin{array}{c}
F_{h} \\
-v_{e}
\end{array}\right]=\left[\begin{array}{ll}
h_{11} & h_{12} \\
h_{21} & h_{22}
\end{array}\right] \cdot\left[\begin{array}{l}
v_{h} \\
F_{e}
\end{array}\right]
$$

This Raisbeck passivity criterion consists of the following conditions:

- no $h_{i j}$ has poles in the right half plane,

- any poles of the $h$-parameters $h_{11}, h_{12}, h_{21}$ and $h_{22}$ on the imaginary axis are simple, and the residues $d_{11}, d_{12}, d_{21}$ and $d_{22}$ at these poles satisfy the conditions: $d_{11} \geq 0, d_{22} \geq 0$ and $d_{11} d_{22}-d_{12} d_{21} \geq 0$ with $d_{21}=d_{12}^{*}$, and

- the real $\left(r_{i j}=\Re\left(h_{i j}\right)\right)$ and imaginary $\left(i_{i j}=\Im\left(h_{i j}\right)\right)$ parts of the $h_{i j^{-}}$ elements satisfy:

$$
\begin{gathered}
r_{11} \geq 0, \quad r_{22} \geq 0, \forall \omega \\
4 r_{11} r_{22}-\left(r_{12}+r_{21}\right)^{2}-\left(i_{12}-i_{21}\right)^{2} \geq 0, \forall \omega .
\end{gathered}
$$

\footnotetext{
${ }^{4} \mathrm{An}$ immittance matrix is a way to represent a two-port network. Depending on the choice of the network input and output, the immittance matrix is an impedance, an admittance, a hybrid or an inverse hybrid matrix Hannaford 1989a, Haykin, 1970. In this paper, the hybrid matrix is used.
}

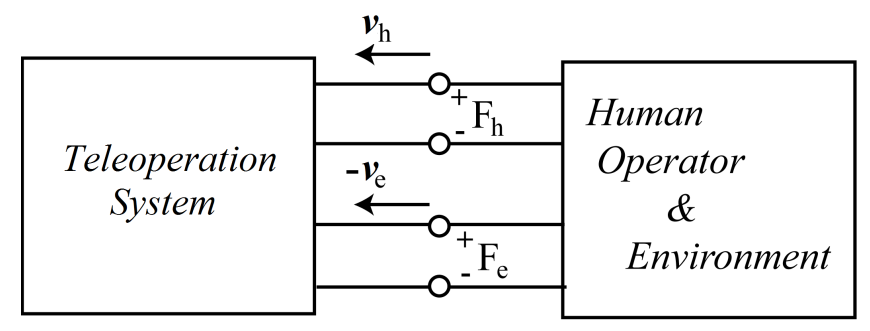

Figure 3: A two-port network representation of a teleoperation system 
In the case of the Position-Force controller, the hybrid matrix is found to be:

$$
\mathbf{H}_{\mathbf{P}-\mathbf{F}}=\left[\begin{array}{cc}
M_{m} s+B_{m} & \lambda \\
\frac{-\mu K_{p}}{M_{s} s^{2}+\left(B_{s}+K_{v}\right) s+K_{p}} & \frac{s}{M_{s} s^{2}+\left(B_{s}+K_{v}\right) s+K_{p}}
\end{array}\right]
$$

for the P-F scheme, whereas the PD-F scheme leads to the following hybrid matrix:

$$
\mathbf{H}_{\mathbf{P D}-\mathbf{F}}=\left[\begin{array}{cc}
M_{m} s+B_{m} & \lambda \\
\frac{-\mu\left(K_{v} s+K_{p}\right)}{M_{s} s^{2}+\left(B_{s}+K_{v}\right) s+K_{p}} & \frac{s}{M_{s} s^{2}+\left(B_{s}+K_{v}\right) s+K_{p}}
\end{array}\right]
$$

For both hybrid matrices, it is relatively straightforward to see that all above mentioned conditions, except 111, hold. After replacing $s$ by $j \omega$ and performing some algebraic manipulations, (11) can be written as:

$$
-\lambda^{2} M_{s}^{2} \omega^{4}+a_{2} \omega^{2}-K_{p}(\lambda-\mu)^{2} \geq 0, \forall \omega
$$

with

$$
\begin{aligned}
a_{2}= & 4 B_{m}\left(B_{s}+K_{v}\right)-\lambda^{2}\left(B_{s}+K_{v}\right)^{2}-\mu^{2} K_{v}^{2} \\
& +2 \mu \lambda K_{p} M_{s}(\lambda-\mu)+2 \mu \lambda K_{v}\left(B_{s}+K_{v}\right)
\end{aligned}
$$

for the PD-F scheme. With $x=\omega^{2}$, this condition is of the from $a x^{2}+b x+c \geq$ $0, \forall x \geq 0$ and Appendix A explains how to interpret such condition. One can verify that this inequality in $\omega$ can only hold in the trivial case that $M_{s}=B_{s}=$ 0 . Note that a similar derivation can be made for the $\mathbf{P}-\mathbf{F}$ scheme. The above derivation allows the formulation of the following theorem:

THEOREM 1 (Active Position-Force Controller) The Position-Force controllers defined by (6) and (7) or by (6) and (8) form a non-passive two-port system when applied upon the teleoperator (3) and (4) for non-trivial parameters of the system.

As mentioned in the introduction, 2-port passivity is a conservative property as it takes into account direct interaction between operator and environment. The next section analyzes the absolute stability property which is a less conservative property incorporating the knowledge that no direct interaction occurs between the operator and the environment.

\section{Absolute Stability Analysis}

A linear two-port is absolutely or unconditionally stable if and only if there is no set of passive one-ports for which the coupled system is unstable. To evaluate the absolute stability of a teleoperation system, one can look at the structured singular value Colgate, 1993, Vander Poorten et al., 2006 or make use of Llewellyn's stability criteria |Llewellyn, |1952]. The latter is used here and has been used by a.o. Adams and Hannaford 1999, Hashtrudi-Zaad and Salcudean 2001] and Cho and Park 2005. Llewellyn's criteria are equivalent to the following conditions in terms of the immittance matrix $P$ :

- the immittance parameters $p_{11}$ and $p_{22}$ have no poles in the open righthalf-plane (RHP), 
- any poles of $p_{11}$ and $p_{22}$ on the imaginary axis are simple and have real and positive residues, and

- the inequalities

$$
\begin{gathered}
\Re\left(p_{11}\right) \geq 0, \forall \omega \\
\eta(\omega)=-\cos \left(\angle p_{12} p_{21}\right)+2 \frac{\Re\left(p_{11}\right) \Re\left(p_{22}\right)}{\left|p_{12} p_{21}\right|} \geq 1, \forall \omega .
\end{gathered}
$$

These conditions are valid for any member of the immittance class. Here, the conditions are again checked for the hybrid matrices $(12)$ and $(13)$. For both hybrid matrices, it is relatively straightforward to see that all conditions, except for inequality (17), hold. Therefore, the following subsections elaborate further on inequality (17) for both the P-F and the PD-F scheme.

\subsection{Absolute stability of the P-F scheme}

The parameter $\eta(\omega)$ in inequality (17) contains two individual terms, which can be written as $\eta_{1}(\omega)$ and $\eta_{2}(\omega)$. For the $\mathbf{P}-\mathbf{F}$ scheme these two parameters become:

$$
\begin{array}{r}
\eta_{1}(\omega)=\cos \left(\angle \frac{\mu \lambda K_{p}}{M_{s} s^{2}+\left(B_{s}+K_{v}\right) s+K_{p}}\right) \\
\eta_{2}(\omega)=2 \frac{\Re\left(M_{m} s+B_{m}\right) \Re\left(\frac{s}{M_{s} s^{2}+\left(B_{s}+K_{v}\right) s+K_{p}}\right)}{\left|\frac{-\mu \lambda K_{p}}{M_{s} s^{2}+\left(B_{s}+K_{v}\right) s+K_{p}}\right|}
\end{array}
$$

Replacing $s$ by $j \omega$ results in the following analytic equations:

$$
\begin{gathered}
\eta_{1}(\omega)=\frac{K_{p}-M_{s} \omega^{2}}{\sqrt{\left(K_{p}-M_{s} \omega^{2}\right)^{2}+\left(B_{s}+K_{v}\right)^{2} \omega^{2}}} \\
\eta_{2}(\omega)=\frac{2 B_{m}\left(B_{s}+K_{v}\right) \omega^{2}}{\mu \lambda K_{p} \sqrt{\left(K_{p}-M_{s} \omega^{2}\right)^{2}+\left(B_{s}+K_{v}\right)^{2} \omega^{2}}}
\end{gathered}
$$

Based on these analytic equations, it can be derived that the condition (17), i.e. $\eta_{1}(\omega)+\eta_{2}(\omega) \geq 1, \forall \omega$, only holds when the parameters of the system satisfy both the following conditions:

$$
\begin{array}{r}
\mu \lambda \leq 4 \frac{B_{m}}{\left(B_{s}+K_{v}\right)}, \\
\mu \lambda \leq \frac{B_{m}\left(B_{s}+K_{v}\right)}{M_{s} K_{p}} .
\end{array}
$$

Fig. 4illustrates these conditions for the parameters of Table 1. For these parameters, the maximum value of $\mu \lambda$ is 0.127 . All sets of parameters that belong to the shaded area will result in an absolutely stable system. The maximum value of $\mu \lambda$ can be found by multiplying the inequalities (22) and (23):

$$
(\mu \lambda)^{2} \leq \frac{4 B_{m}}{\left(B_{s}+K_{v}\right)} \cdot \frac{B_{m}\left(B_{s}+K_{v}\right)}{M_{s} K_{p}} \Rightarrow \mu \lambda^{\max } \leq \frac{2 B_{m}}{\sqrt{M_{s} K_{p}}}
$$

This means that the absolute maximum value of $\mu \lambda$ can be reached for a critically damped slave $\left(B_{s}+K_{v}=2 \sqrt{M_{s} K_{p}}\right)$, i.e. for the parameters of Table 1 


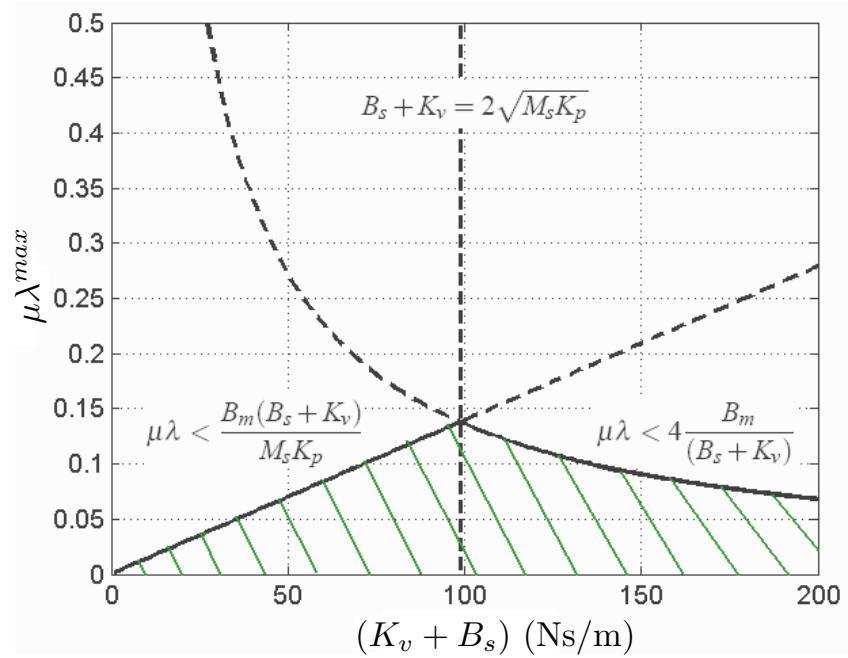

Figure 4: The absolute stability conditions for the P-F scheme, expressed as an upper boundary on the scaling factors $\mu \lambda$ for constant values of $B_{m}, K_{p}$ and $M_{s}$. The dashed area is the region of absolute stability.

$K_{v}=87.8 \mathrm{~N} / \mathrm{m}$ and $\mu \lambda^{\max }=0.137$. Note that Bu et al. [1996] found the same maximum value of $\mu \lambda$ for a critically damped slave by looking at closed loop stability. However, their analysis only covers the case for a critically damped slave, where the analysis presented here also describes the behaviour of non-critically damped slave robots. The above results are summarized in the following theorem:

THEOREM 2 (Absolutely stable P-F controller) The Position-Force controller without master velocity-feedforward, applied upon the teleoperator (3), (4) and (5), and expressed by control laws (6) and (8) is absolutely stable when the scale factors $\lambda$ and $\mu$ respect both inequalities (22) and (23).

Fig. 5 a shows the parameters $\eta(\omega), \eta_{1}(\omega)$ and $\eta_{2}(\omega)$ for two different values of the scaling factors $\mu \lambda$ and the parameters of Table 1. For $\mu \lambda=0.1$, i.e. a value that satisfies the conditions $(22)$ and $(23)$, one can see that $\eta(\omega) \geq 1$ for all frequencies. Thus, the system with $\mu \lambda=0.1$ is absolutely stable.

\subsection{Absolute stability of the PD-F scheme}

For the PD-F scheme, the parameter $\eta(\omega)$ can again be written as the sum of $\eta_{1}(\omega)$ and $\eta_{2}(\omega)$, with:

$$
\begin{aligned}
& \eta_{1}(\omega)=\cos \left(L \frac{\mu \lambda\left(K_{v} s+K_{p}\right)}{M_{s} s^{2}+\left(B_{s}+K_{v}\right) s+K_{p}}\right) \\
& \eta_{2}(\omega)=2 \frac{\Re\left(M_{m} s+B_{m}\right) \Re\left(\frac{s}{M_{s} s^{2}+\left(B_{s}+K_{v}\right) s+K_{p}}\right)}{\left|\frac{-\mu \lambda\left(K_{v} s+K_{p}\right)}{M_{s} s^{2}+\left(B_{s}+K_{v}\right) s+K_{p}}\right|}
\end{aligned}
$$



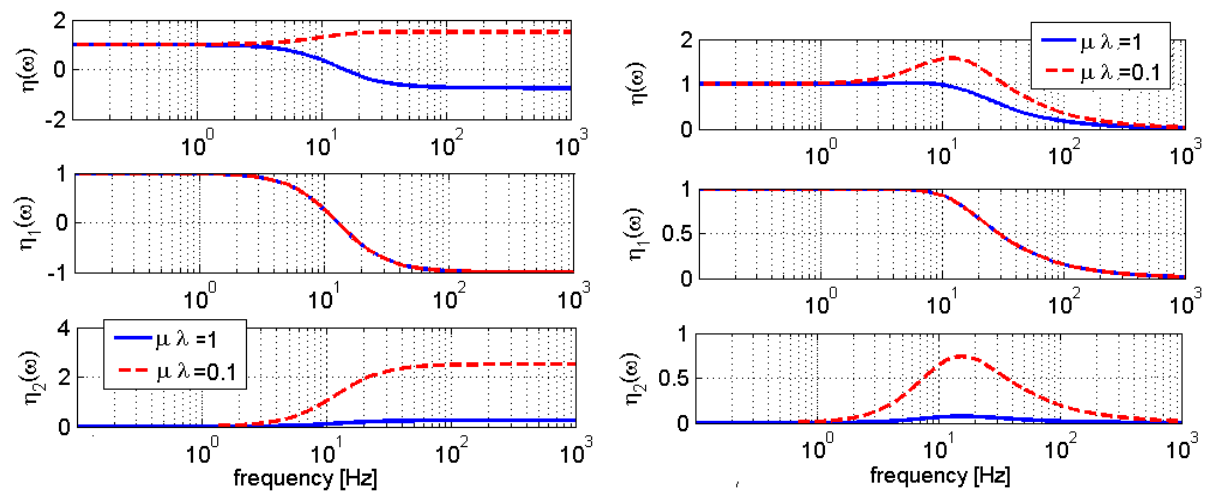

(a) the P-F scheme

(b) the PD-F scheme

Figure 5: The parameters $\eta(\omega), \eta_{1}(\omega)$ and $\eta_{2}(\omega)$ for two different values of $\mu \lambda$ and the parameters of Table 1. Only in case of the P-F scheme with $\mu \lambda=0.1$, the condition for absolute stability, i.e. $\eta(\omega) \geq 1, \forall \omega$ holds.

Replacing $s$ by $j \omega$ results in the following analytic equations:

$$
\begin{gathered}
\eta_{1}(\omega)=\operatorname{sign}(\mu \lambda) \frac{K_{p}^{2}+\left(K_{v}^{2}+K_{v} B_{s}-M_{s} K_{p}\right) \omega^{2}}{\sqrt{\left(K_{p}-M_{s} \omega^{2}\right)^{2}+\left(\left(B_{s}+K_{v}\right) \omega\right)^{2}} \sqrt{K_{p}^{2}+K_{v}^{2} \omega^{2}}} \\
\eta_{2}(\omega)=\frac{2 B_{m}\left(B_{s}+K_{v}\right) \omega^{2}}{|\mu \lambda| \sqrt{\left(K_{p}-M_{s} \omega^{2}\right)^{2}+\left(\left(B_{s}+K_{v}\right) \omega\right)^{2}} \sqrt{K_{p}^{2}+K_{v}^{2} \omega^{2}}}
\end{gathered}
$$

From these equations follows that for $\omega \rightarrow \infty, \eta_{1}(\omega) \rightarrow 0$ and $\eta_{2}(\omega) \rightarrow 0$, independent of the parameters of the system. This means that $(17)$ never holds for all $\omega$, leading to the following result:

THEOREM 3 (Non absolutely stable PD-F controller) The PositionForce controller with master velocity-feedforward, applied upon the teleoperator (3), (4) and (5), and expressed by control laws (6) and by (7) is never absolutely stable when $M_{s} \neq 0$.

Tavakoli et al. 2007. made a similar conclusion based on an analytic analysis of the Llewellyn's stability criteria. However, their conclusion is less general as their model does not include master damping $B_{m}$, which plays a crucial role in the stability of the Position-Force controller. In the case of the P-F scheme e.g., it is shown that master damping is mandatory to obtain an absolutely stable system. Fig. $5 \mathrm{~b}$ shows $\eta(\omega), \eta_{1}(\omega)$ and $\eta_{2}(\omega)$ for two values of the scaling factors $\mu \lambda$ and the parameters of Table 1 . One can see that for both values of $\mu \lambda, \eta(\omega)<1$ over a large range of frequencies, which means that the system is not absolutely stable for these values of $\mu \lambda$. 
The above derivations show that stable interaction can be achieved with any passive environment for the Position-Force controller, but only for very limited values of $\mu \lambda$ in the case of $\mathbf{P - F}$ and even never in the case of PD-F. These conclusions are illustrations of the fact, mentioned in the introduction, that 2-port passivity and absolute stability can result in no or very restricting conditions. The following section investigates how the incorporation of knowledge on the environment can result in more interesting conditions.

\section{Bounded Environment Passivity Analysis}

This section describes and applies the Bounded Environment Passivity method, proposed by Willaert et al. [2009]. The method also originates from the passivity concept, but in contrast to two-port passivity and absolute stability, the combined dynamics of the master, slave, controller and environment are analyzed. These dynamics represent the one-port network $Y_{M S}$ and, as shown in Fig 6 . this is the one-port network the human operator interacts with. Passivity of this one-port network is a sufficient condition for coupled stability with any passive operator and can be checked by verifying the positive-realness of $Y_{M S}$ Colgate and Hogan, 1988. The admittance $Y_{M S}$ is positive real if, in addition to being real for real $s$, it fulfils the following requirements Haykin, 1970:

- $Y_{M S}$ has no poles in the right half of the complex-frequency plane

- Any poles of $Y_{M S}$ on the imaginary axis are simple with real and positive residues.

- $\Re\left(Y_{M S}\right) \geq 0$ for all $\omega$, where $\Re$ denotes the real part of $Y_{M S}$.

The first two requirements deal with the uncoupled stability of $Y_{M S}$, while the positive real part requirement deals with the coupled stability of $Y_{M S}$. Proving BIBO-stability of $Y_{M S}$ is a sufficient condition for the first two requirements and this can be done using the Routh-Hurwitz criterion.

In order to determine $Y_{M S}$, an assumption has to be made about the environment $Z_{e}$. As stated in the introduction, pure springs and pure masses can be considered as the worst case environments since their admittance is not strictly passive. Therefore, the Bounded Environment Passivity method analyses the positive-realness of $Y_{M S}$ for a pure mass $\left(Z_{e}=M_{e} s\right)$ and a pure stiffness

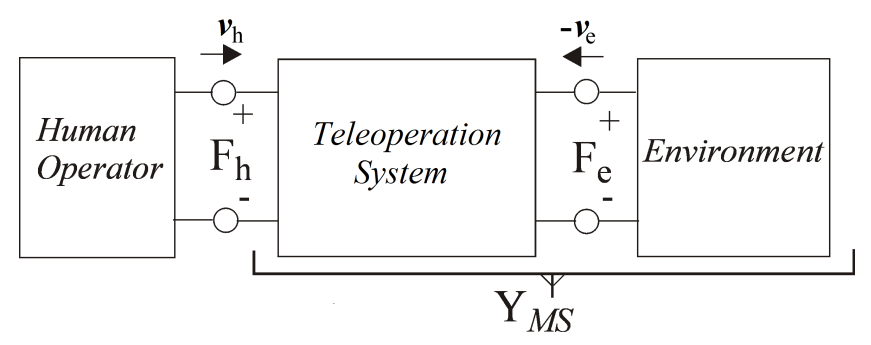

Figure 6: A one-port network $Y_{M S}$ representation of a combined teleoperatorenvironment system. 


$$
\begin{aligned}
& \left(Z_{e}=\frac{K_{e}}{s}\right) \text { as environment, both with a bounded magnitude: } \\
& Y_{M S\left(M_{e}\right)} \text { BIBO-stable and } \Re\left(Y_{M S\left(M_{e}\right)}\right) \geq 0 \text { with } M_{e} \in\left[0, M_{e}^{\max }\right], \\
& Y_{M S\left(K_{e}\right)} \text { BIBO-stable and } \Re\left(Y_{M S\left(K_{e}\right)}\right) \geq 0 \text { with } K_{e} \in\left[0, K_{e}^{\max }\right] .
\end{aligned}
$$

To extend the results of these two analyses, the following assumption is made:

ASSUMPTION 1 (Extension to mass-spring-damper systems) If $Y_{M S}$ is passive for all pure masses $M_{e} \in\left[0, M_{e}^{\max }\right]$ and all pure stiffnesses $K_{e} \in$ $\left[0, K_{e}^{\max }\right], Y_{M S}$ is guaranteed to be passive for all environments $Z_{e}=M_{e} s+$ $B_{e}+\frac{K_{e}}{s}$ with $M_{e}<M_{e}^{\max }, K_{e}<K_{e}^{\max }$ and $B_{e} \in[0, \infty]$.

This assumption has not been proven at this point, neither has a counterexample been found despite extensive numerical simulations. The validity of this assumption for the PD-F scheme and the parameters of Table 1 is illustrated in Appendix D. As this assumption has the potential to largely extend the usability of the Bounded Environment Passivity method, a rigorous proof for it is an important topic for further research.

In the remainder of this paper, the considered environment is a pure stiffness. The motivation behind this work is telesurgery and one can assume that the inertial properties of the environment the surgeon interacts with, are negligible. Even more important is the fact, proven in Appendix $B$, that a pure mass cannot make the admittance $Y_{M S}$ active, i.e. $M_{e}^{\max }=\infty$. As a consequence, a pure mass as environment does not yield any stability problems. Furthermore, for the application of telesurgery, it is relatively easy to delineate a range of stiffnesses $\left[0, K_{e}^{\max }\right]$ the surgeon typically interacts with. Assuming a pure stiffness as environment, the admittance $Y_{M S}$ can be written as:

$$
Y_{M S\left(K_{e}\right)}=\frac{s\left(M_{s} s^{2}+\left(B_{s}+K_{v}\right) s+\left(K_{p}+K_{e}\right)\right)}{\left(M_{m} s^{2}+B_{m} s\right)\left(M_{s} s^{2}+\left(B_{s}+K_{v}\right) s+\left(K_{p}+K_{e}\right)\right)+\mu \lambda K_{e} K_{p}},
$$

for the $\mathbf{P}-\mathbf{F}$ scheme and as:

$$
Y_{M S\left(K_{e}\right)}=\frac{s\left(M_{s} s^{2}+\left(B_{s}+K_{v}\right) s+\left(K_{p}+K_{e}\right)\right)}{\left(M_{m} s^{2}+B_{m} s\right)\left(M_{s} s^{2}+\left(B_{s}+K_{v}\right) s+\left(K_{p}+K_{e}\right)\right)+\mu \lambda K_{e}\left(K_{v} s+K_{p}\right)},
$$

for the PD-F scheme. As mentioned above, checking the positive-realness of these admittances includes verifying BIBO-stability of $Y_{M S\left(K_{e}\right)}$ and verifying whether the real part of $Y_{M S\left(K_{e}\right)}$ is positive. From our observation, the BIBOstability condition for these admittances is always less restricting than the positive real part condition. Therefore, for reasons of brevity, only the positive real part conditions are mentioned here $5^{5}$

Verifying whether the real part of these admittances is positive results in analytic and/or numerical conditions, depending on the complexity of the condition $\Re\left(Y_{M S}\right) \geq 0$ for all $\omega$. These conditions can be presented as limits to the achievable scale factors $\lambda \mu$ as a function of the range of $K_{e}$ and as a function of hardware and control parameters. Conversely, these conditions can also be presented as a limit on the environment stiffness in function of all hardware and

\footnotetext{
${ }^{5}$ Note that the BIBO-stability of a real setup, i.e. the uncoupled stability, can be verified easily, while verifying the coupled stability, i.e. the interaction with all possible passive operators, is practically not feasible.
} 


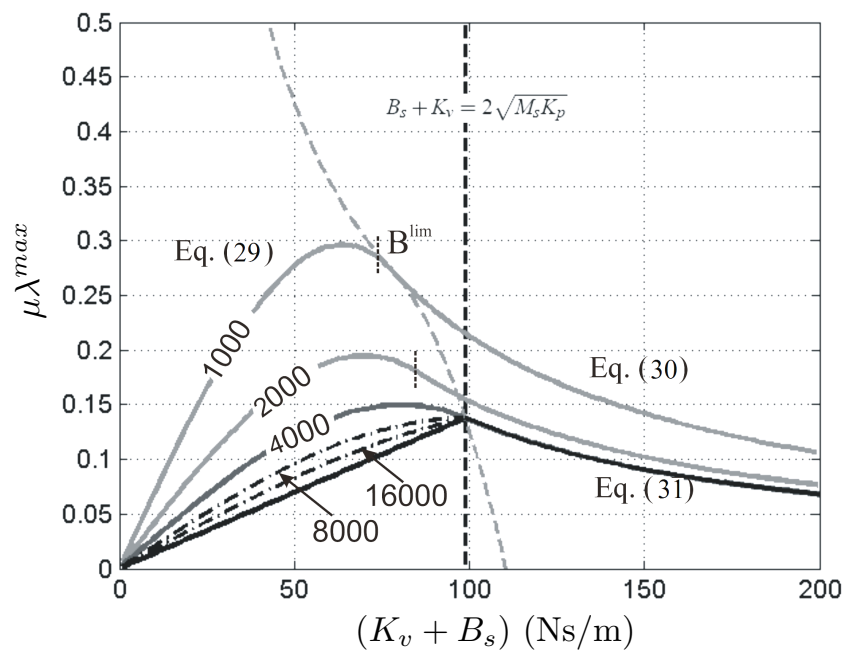

Figure 7: The upper boundary on the scaling factors $\mu \lambda$ for different values of $K_{e}^{\max }(1000-\infty \mathrm{N} / \mathrm{m})$, expressed as a function of the sum of the control damping $K_{v}$ and the hardware damping $B_{S}$ for the $\mathbf{P}-\mathbf{F}$ scheme. Note that $K_{p}$, the stiffness of the position controller of the slave, is $4000 \mathrm{~N} / \mathrm{m}$.

control parameters, including the scaling factors. Furthermore, these conditions can be used as design guidelines for the hardware and as tuning guidelines for the controllers. Hereunder, conditions are derived first for the $\mathbf{P}-\mathbf{F}$, then for the PD-F scheme.

\subsection{Bounded Environment passivity for the P-F scheme}

The positive real part condition for the $\mathbf{P}-\mathbf{F}$ scheme can be checked analytically. Following the approach described in Appendix A and after replacing $s$ by $j \omega$, the condition $\Re\left(Y_{M S\left(K_{e}\right)} \geq 0\right.$ can be expressed as:

$$
\begin{gathered}
\left(M_{s}^{2} B_{m}\right) \omega^{6}+\left(B_{m}\left(B_{s}+K_{v}\right)^{2}-2\left(K_{p}+K_{e}\right) M_{s} B_{m}\right) \omega^{4}+ \\
\left(B_{m}\left(K_{p}+K_{e}\right)^{2}-\mu \lambda K_{e} K_{p}\left(B_{s}+K_{v}\right)\right) \omega^{2} \geq 0, \forall \omega \geq 0,
\end{gathered}
$$

which results in the following conditions on the system parameters:

$$
\begin{aligned}
& B^{l i m}=\min \left[\sqrt{2\left(K_{p}+K_{e}^{\text {max }}\right) M_{s}}, 2 \sqrt{K_{p} M_{s}}\right] \\
& 0 \leq\left(B_{s}+K_{v}\right) \leq B^{l i m} \quad: \quad \mu \lambda \leq \frac{B_{m}\left(B_{s}+K_{v}\right)}{M_{s} K_{p}}\left[1+\frac{K_{p}}{K_{e}^{m a x}}-\frac{\left(B_{s}+K_{v}\right)^{2}}{4 K_{e}^{m a x} M_{s}}\right] \\
& \left(B_{s}+K_{v}\right) \geq B^{l i m} \text { and } K_{e}^{m a x} \leq K_{p} \quad: \quad \mu \lambda \leq \frac{B_{m}\left(K_{p}+K_{e}^{m a x}\right)^{2}}{\left(B_{s}+K_{v}\right) K_{p} K_{e}^{\max }} \\
& \left(B_{s}+K_{v}\right) \geq B^{l i m} \text { and } K_{e}^{\text {max }}>K_{p} \quad: \quad \mu \lambda \leq \frac{4 B_{m}}{\left(B_{s}+K_{v}\right)}
\end{aligned}
$$

Fig. 7]illustrates these conditions for the values in Table 1 and for different values of the selected maximum environment stiffness $K_{e}^{\max }(1000, \ldots, 16000 \mathrm{~N} / \mathrm{m})$. The parameters belonging to the area below the curves result in a passive admittance $Y_{M S\left(K_{e}\right)}$. The maximum attainable value $\lambda \mu^{\max }$ for a given $K_{e}^{\max }$ is reached at different values of $B_{s}+K_{v}$. For $K_{e}^{\max }<2 K_{p}$, the maximum attainable value $\lambda \mu^{\max }$ is:

$$
\mu \lambda^{\max }=\frac{4}{3} \frac{B_{m}}{\sqrt{M_{s}} K_{p} K_{e}^{\max }} \sqrt{\frac{\left(K_{e}^{\max }+K_{p}\right)^{3}}{3}} .
$$


for the following value of $B_{s}+K_{v}$ :

$$
\left(B_{s}+K_{v}\right)^{\max }=\sqrt{\frac{4 M_{s}\left(K_{e}^{\max }+K_{p}\right)}{3}},
$$

When $K_{e}^{\max } \geq 2 K_{p}$, the maximal $\mu \lambda$ value is reached for critical damping of the slave and equals $2 B_{m} / \sqrt{M_{s} K_{p}}$ (this follows from $35,,(39)$ and $(38)$ ). Note that this value corresponds to the value found for absolute stability in subsection 4.1. Thus, no gain in the factor $\mu \lambda$ can be achieved for the P-F scheme by using Bounded Environment Passivity when $K_{e}^{\max } \geq 2 K_{p}$, while for smaller

$K_{e}^{\max }$, the factor $\mu \lambda$ can be increased: for $K_{e}^{\max }=1000 \mathrm{~N} / \mathrm{m}$ e.g., $\mu \lambda^{\max }$ is 0.296 instead of 0.137 . The latter is the maximum value for $\mu \lambda$ that can be reached under the condition of absolute stability.

Based on (38) the effect of different hardware and controller parameters on $\mu \lambda^{\max }$ is calculated and plotted in Fig. 8a. This figure shows the relation between the selected maximum environment stiffness $K_{e}^{\max }$ and $\mu \lambda^{\max }$ as well as the effect of $M_{s}, B_{m}$ and $K_{p}$ on $\mu \lambda^{\max }$ for a fixed maximum environment stiffness $K_{e}^{\max }=1000 \mathrm{~N} / \mathrm{m}$. One can see for:

- $K_{e}^{\max }$ : a significant increase in allowable $\mu \lambda$ can only be obtained when the maximum environment stiffness $K_{e}^{\max }$ is limited. This finding is consistent with the generally known problem that the Position-Force controller faces stability problems when contacting stiff environments. Note that for $K_{e}^{\max } \rightarrow \infty$, the curve evolves towards the condition of absolute stability (derived in subsection 4.1).

- $M_{s}$ : for a slave with a lower inertia, larger values of $\mu \lambda$ are allowed. This is an important design guideline.

- $K_{p}$ : a tighter position loop at the slave is found to increase the maximal $\mu \lambda$ value. This is an example of a tuning guideline. Note, that $K_{p}$ is typically practically limited in order to guarantee closed-loop stability of the position controller of the slave. For very small values of $K_{p}$ the maximal $\mu \lambda$ value is increasing for decreasing values of $K_{p}$, this should be no surprise as for $K_{p} \rightarrow 0$, the master-slave link is opened. The importance of this finding is thus minimal.

- $B_{m}$ : an increase of master damping allows larger $\mu \lambda$ values. Note that extra damping at the master jeopardizes the feeling the human operator has when moving in free space. Consequently, a compromise should be found.

- the parameters of Table1, i.e. with $\mu=\lambda=1$, the maximum environment stiffness $K_{e}^{\max }$ for which $Y_{M S\left(K_{e}\right)}$ is passive is $161 \mathrm{~N} / \mathrm{m}$.

\subsection{Bounded Environment passivity for the PD-F scheme}

In the same way as done for the $\mathbf{P}-\mathbf{F}$ scheme, the positive real part condition for the PD-F scheme can be expressed as:

$$
\begin{aligned}
& \left.\left(M_{s}^{2} B_{m}\right) \omega^{6}+\left(B_{m}\left(B_{s}+K_{v}\right)^{2}-2\left(K_{p}+K_{e}\right) M_{s} B_{m}-\mu \lambda M_{s} K_{v} K_{e}\right)\right) \omega^{4} \\
& +\left(B_{m}\left(K_{p}+K_{e}\right)^{2}+\mu \lambda K_{v} K_{e}^{2}-\mu \lambda K_{e} K_{p} B_{s}\right) \omega^{2} \geq 0, \quad \forall \omega \geq 0 .
\end{aligned}
$$




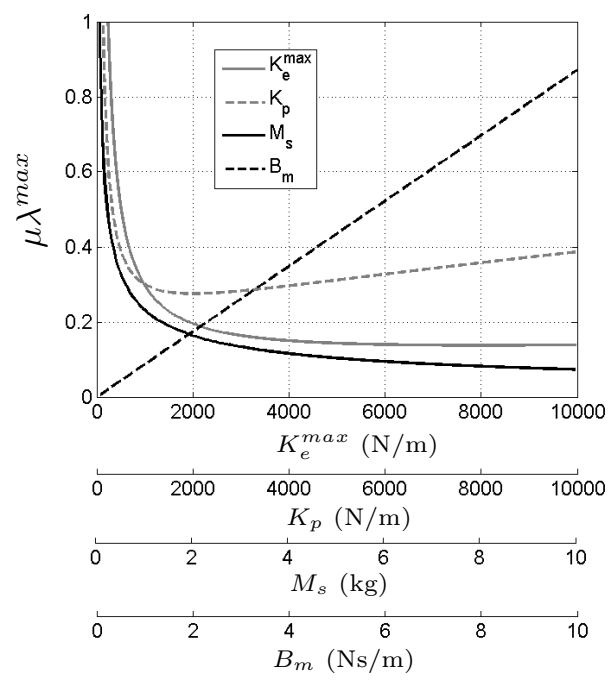

(a) the P-F scheme

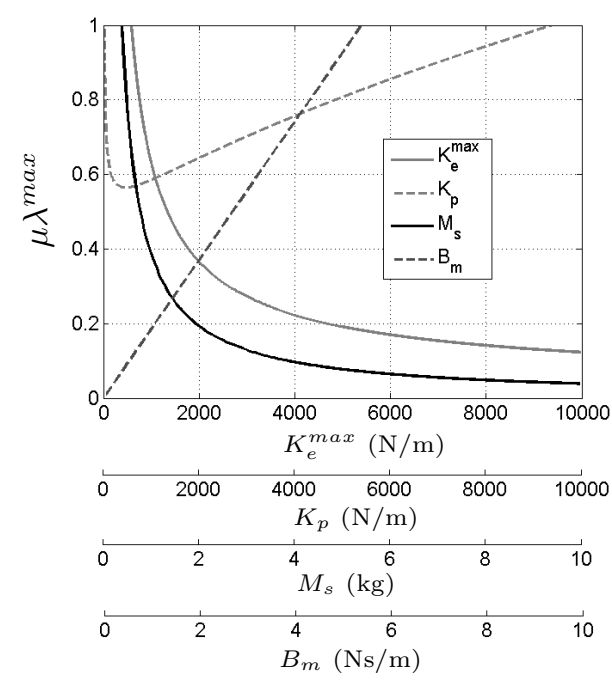

(b) the PD-F scheme

Figure 8: Effect of $K_{e}^{\max }$ on $\mu \lambda^{\max }$ and the effect of $M_{s}, B_{m}$ and $K_{p}$ on $\mu \lambda^{\max }$ when $K_{e}^{\max }=1000$ for the parameters of Table 1 .

Again, this condition can be reformulated as a set of conditions upon the parameters. Here, these conditions are rather complicated and can be found in the Appendix C For $K_{v} \leq\left(M_{s} K_{p}\right) / B_{s}$, the conditions simplify to one condition that can be expressed as a maximum for $\mu \lambda$ :

$$
\mu \lambda \leq \frac{B_{m}\left(B_{s}+K_{v}\right)}{M_{s} K_{e} K_{v}^{2}}\left(\left(B_{s}+K_{v}\right) K_{v}-2 M_{s} K_{p}+2 \sqrt{M_{s}^{2} K_{p}^{2}+M_{s} K_{v}^{2} K_{e}-K_{p} K_{v} B_{s} M_{s}}\right) .
$$

Fig. 9 illustrates this relation for the parameters of Table 1 and different values of $K_{e}^{\max }(1000, \ldots, 27000 \mathrm{~N} / \mathrm{m})$. In order to compare the PD-F scheme with the P-F scheme, the curves delimiting the region of Bounded Environment Passivity for the P-F scheme are shown again. For relatively low values of $K_{e}^{\max }$ the PD$\mathbf{F}$ scheme outperforms the $\mathbf{P}-\mathbf{F}$ scheme. For a maximum environment stiffness $K_{e}^{\max }=1000 \mathrm{~N} / \mathrm{m}$ and $K_{v}=88.7 \mathrm{Ns} / \mathrm{m}{ }^{6}$ e.g., $\mu \lambda=0.67$ (compared to 0.137 and 0.296 based on respectively absolute stability and Bounded Environment Passivity of the P-F scheme).

Based on (41), Fig. 8b shows the effect on the maximum allowed $\mu \lambda^{\max }$ of the selected $K_{e}^{\max }$ as well as the effect of $M_{s}, B_{m}$ and $K_{p}$ on $\mu \lambda^{\max }$ for a fixed maximum environment stiffness $K_{e}^{\max }=1000 \mathrm{~N} / \mathrm{m}$. Note that the relation $K_{p}-\mu \lambda$ shown here, supposes a fixed damping ratio of the slave, i.e. $M_{s}$ is kept constant while $K_{v}$ follows $B_{s}+K_{v}=2 \sqrt{M_{s} K_{p}}$. Similar relations as in Fig. 8a appear for the dependency on $K_{e}^{\max }, M_{s}, B_{m}$ and $K_{p}$. The three main differences are:

- For $K_{e}^{\max } \rightarrow \infty$, the curve showing the effect of $K_{e}^{\max }$ on $\mu \lambda$ evolves towards zero, which corresponds the conclusion in subsection 4.2 that the PD-F scheme can never be absolutely stable.

${ }^{6}$ This corresponds to critical damping of the slave: $B_{s}+K_{v}=2 \sqrt{M_{s} K_{p}}$ 


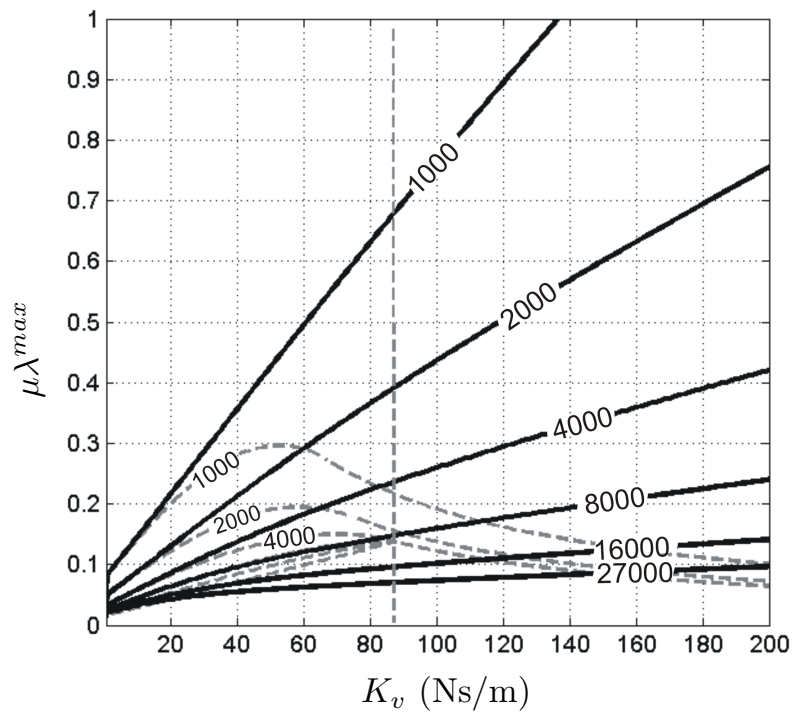

Figure 9: The upper boundary on the scaling factors $\mu \lambda$ for different values of $K_{e}^{\max }(1000-27000 \mathrm{~N} / \mathrm{m})$, expressed as a function of the control damping $K_{v}$ for both the P-F (dashed) and the PD-F scheme (solid).

- Although the PD-F scheme cannot be absolutely stable, while the P-F scheme can, the PD-F scheme allows significantly higher values of $\mu \lambda$ for a certain value of $K_{e}^{\max }, M_{s}, B_{m}$ and $K_{p}$.

- For the parameters of Table 1 , i.e. with $\mu=\lambda=1$ the maximum environment stiffness $K_{e}^{\max }$ is $580 \mathrm{~N} / \mathrm{m}$ instead of $161 \mathrm{~N} / \mathrm{m}$.

\section{The effect of non-idealities}

So far, 2-port passivity, absolute stability and Bounded Environment Passivity have been studied for an ideal controller and an ideal master and slave modelled as rigid-bodies. However, a real teleoperation system can suffer from non-idealities like communication delays, filters, flexibilities, backlash and friction. Based on Bounded Environment Passivity, this section analyzes the effect of two of these non-idealities which apply especially in the case of telesurgery.

\subsection{A low-pass filter in the loop}

In literature, the use of low-pass filters for elimination of surgical hand tremor is often mentioned as one of the benefits of telesurgery Okamura, 2004, Hockstein et al., 2007. Furthermore, low-pass filters can also be used to avoid excitation of the structural resonance frequencies of the slave. However, one should realize that such low-pass filters can jeopardize the quality of haptic feedback. Although Daniel and McAree 1998 suggest that for the P-F scheme a low-pass filtering in the loop improves the stability properties, on the contrary it is shown here that such a low-pass filter has a very negative effect on the passivity of $Y_{M S\left(K_{e}\right)}$ 


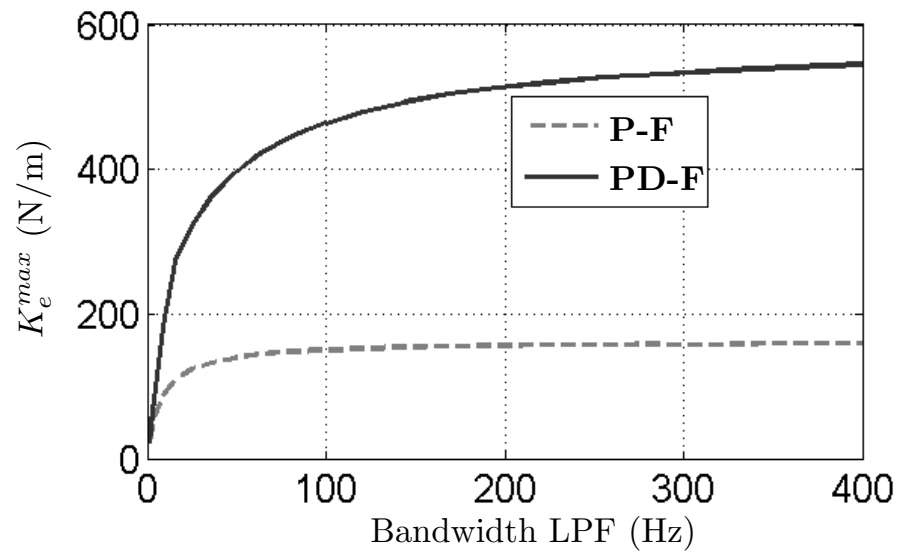

Figure 10: The maximum environment stiffness for which $Y_{M S\left(K_{e}\right)}$ is passive in function of the bandwidth of the low-pass filter in the loop.

for both the P-F scheme and the PD-F scheme. This is further confirmed experimentally in section 8 . Consider adding a $1^{\text {st }}$-order low-pass filter on the position command, i.e. replacing $\mu$ in (7) and (8) as follows:

$$
\mu \rightarrow \frac{\mu}{\tau \cdot s+1}
$$

For the P-F scheme, the admittance $Y_{M S\left(K_{e}\right)}$ can now be written as:

$$
Y_{M S\left(K_{e}\right)}=\frac{s(\tau s+1)\left(M_{s} s^{2}+\left(B_{s}+K_{v}\right) s+\left(K_{p}+K_{e}\right)\right)}{(\tau s+1)\left(M_{m} s^{2}+B_{m} s\right)\left(M_{s} s^{2}+\left(B_{s}+K_{v}\right) s+\left(K_{p}+K_{e}\right)\right)+\mu \lambda K_{e} K_{p}},
$$

and for the PD-F scheme as:

$$
Y_{M S\left(K_{e}\right)}=\frac{s(\tau s+1)\left(M_{s} s^{2}+\left(B_{s}+K_{v}\right) s+\left(K_{p}+K_{e}\right)\right)}{(\tau s+1)\left(M_{m} s^{2}+B_{m} s\right)\left(M_{s} s^{2}+\left(B_{s}+K_{v}\right) s+\left(K_{p}+K_{e}\right)\right)+\mu \lambda K_{e}\left(K_{v} s+K_{p}\right)} .
$$

It is important to note that a low-pass filter on the force feedback, i.e. $\lambda \rightarrow$ $\frac{\lambda}{\tau \cdot s+1}$, results in the same admittance $Y_{M S\left(K_{e}\right)}$ and thus has the same effect as a low-pass filter on the position command. For the above admittances, the positive real part condition, i.e. $\Re\left(Y_{M S}\right) \geq 0$ for all $\omega$, results in a $4^{\text {th }}$ order inequality in $x=\omega^{2}$. Consequently, an analytic analysis becomes too complex. Therefore, here, the Bounded Environment Passivity is analyzed numerically in Matlabß. This is done as follows: starting from $K_{e}=0$, the condition $\Re\left(Y_{M S\left(K_{e}\right)}(j \omega)\right) \geq 0$ is verified for $\omega \in\left[0.1-10^{9} \mathrm{rad} / \mathrm{s}\right]$ for increasing values of $K_{e}$ (increment: $1 \mathrm{~N} / \mathrm{m}$ ). When the condition $\Re\left(Y_{M S\left(K_{e}\right)}(j \omega)\right) \geq 0$ no longer holds, $K_{e}^{\max }$ is found. This is done here for the parameters of Table 1, i.e. with $\mu=\lambda=1$, and different values of the bandwidth of the low-pass filter (bandwidth $\left.=\frac{1}{2 \pi \tau}\right)$. Note that for the parameters of Table $1, K_{e}^{\max }$ is respectively $161 \mathrm{~N} / \mathrm{m}$ and $580 \mathrm{~N} / \mathrm{m}$ for the P-F scheme and the PD-F scheme in case that no low-pass filter is in the loop. The latter is derived in Section 5 .

Fig. 10 shows the result for both the P-F scheme and the PD-F scheme. For very large bandwidths $(>60 \mathrm{~Hz}), K_{e}^{\max }$ is only a little smaller than the maximum environment stiffness in case no low-pass filter is in the loop. But for small 


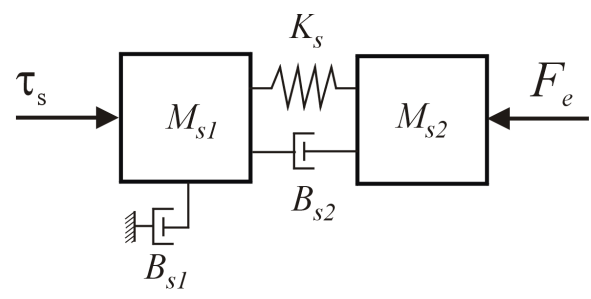

Figure 11: Mechanical model of a flexible slave. $K_{s}$ is the structural stiffness of the slave robot.

bandwidths $(<25 \mathrm{~Hz})$, it is clear that the low-pass filter has a dramatic effect on $K_{e}^{\max }$.

Note that the fundamental stability limits of the Position-Force controller can be intuitively explained by the phase lag introduced by the position controller. As the gain of the position controller $K_{p}$ is practically limited, the position tracking bandwidth is restricted. The conditions derived in section 5 show that increasing $K_{p}$, decreasing $M_{s}$ and adding a velocity feedforward term have a positive effect on the passivity boundaries. This should be no surprise as all of these measures decrease the phase lag of the position controlled slave. Looking at the change of this phase lag, the above mentioned problem with a low-pass filter in the loop, the well-known problem of time-delay in the loop Lawn and Hannaford, 1993, Lawrence, 1993 and the beneficial properties of adding a leadfilter to the control-channel Fite et al. 2001 can be explained.

\subsection{A structural resonance frequency of the slave robot}

It is well known that the presence of link or joint flexibilities can deteriorate the stability properties of force-controlled robots Dwivedy and Eberhard, 2006. Up to now the effect of such flexibilities on transparency and stability received little attention in the domain of teleoperation Christiansson and van der Helm. 2007, Tavakoli and Howe, 2009, although typical master and slave robots, especially the multi-d.o.f. systems, are flexible with the first structural resonance frequency ranging from 5 to $40 \mathrm{~Hz}$. Tavakoli and Howe 2009 study the effect of high-order dynamics of the slave a.o. for a Position-Force controller, but under the assumption of a perfect position controller, i.e. $K_{p}=\infty$. Hereunder, the combination of a realistic controller and a flexible slave is studied. The mechanical model for the slave robot is shown in Fig. 11. For the PD-F scheme, the admittance $Y_{M S\left(K_{e}\right)}$ can now be written as:

$$
Y_{M S\left(K_{e}\right)}=\frac{N u m(s)}{\left(M_{m} s+B_{m}\right)(N u m(s))+\mu \lambda K_{e}\left(B_{s 2} s+K_{s}\right)\left(K_{v} s+K_{p}\right)}
$$

with

$N u m(s)=\left(M_{s 1} s^{2}+\left(B_{s 1}+B_{s 2}+K_{v}\right) s+\left(K_{s}+K_{p}\right)\right) \cdot\left(M_{s 2} s^{2}+B_{s 2} s+\left(K_{s}+K_{e}\right)\right)-\left(B_{s 2} s+K_{s}\right)^{2}$

Note that the analysis is presented only for the the PD-F scheme as the analysis for the P-F scheme results in similar conclusions. Again, the positive real part 


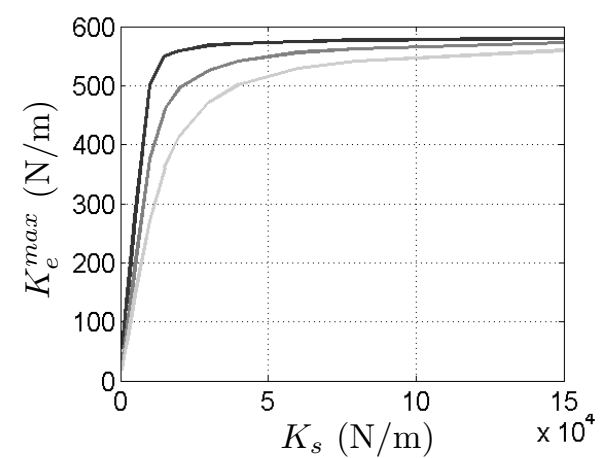

(a)

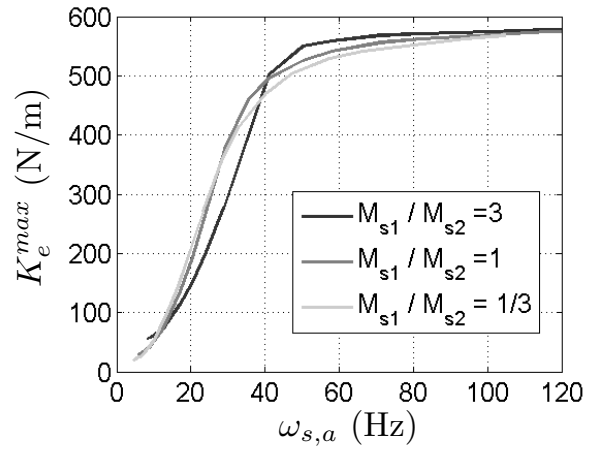

(b)

Figure 12: The maximum environment stiffness for which $Y_{M S\left(K_{e}\right)}$ is passive as a function of (a) the structural stiffness $K_{s}$ of the slave and (b) the structural anti-resonance frequency, $\omega_{s, a}=\sqrt{\frac{K_{s}}{M_{s 2}}}$.

condition for the above admittance is too complex to be analysed analytically. In the same way as described above, the Bounded Environment Passivity is analyzed numerically for the parameters of Table 1 and a whole range of values for the structural stiffness of the slave. Note that the calculation is done for three different mass distributions, while the total mass of the slave is kept constant, i.e. $M_{s 1}+M_{s 2}=0.61 \mathrm{~kg}$.

Fig. 12 shows the result for the PD-F scheme. For high structural antiresonance frequencies $\omega_{s, a}(>50 \mathrm{~Hz})$, the flexibility of the slave has little effect on the maximum environment stiffness, but for low structural anti-resonance frequencies $(<50 \mathrm{~Hz})$, the effect is very clear. This shows that the slave should not only be lightweight (see section 5 ) but also stiff, which is another important design guideline.

The experiments, described in section 8, with a 1 d.o.f.- teleoperation setup show very good correspondence with the conditions derived in section 5 based on a rigid-body model assumption for the master and slave. Although the 1d.o.f. master and slave don't behave as real rigid-bodies, the first resonance frequencies are above $100 \mathrm{~Hz}$. As shown here, the effect of such high structural resonance frequencies is negligible for the Position-Force controller with the parameters of Table 1, which justifies a rigid-body model assumption for this 1 d.o.f.- teleoperation setup.

Note that, for the same 1 d.o.f.- teleoperation setup, also the effect of the $2^{\text {nd }}$ order low-pass filter on the velocity with a bandwidth of $80 \mathrm{~Hz}$ is negligible for both the P-F and the PD-F scheme. The effect of this non-ideality is not explicitly described in this paper, but a similar analysis to the two analyses described above shows that the effect of such a low-pass filter on the passivity of $Y_{M S\left(K_{e}\right)}$ is much smaller than the effect of a low-pass filter on the position or the effect of a structural resonance frequency. Only for bandwidths smaller than $10 \mathrm{~Hz}$, the effect of such a low-pass filter becomes apparent. 


\section{Transparency}

Although this paper focusses on the stability properties of the Position-Force controller, transparency should not be neglected. In the former sections, the admittance $Y_{M S}$ has been calculated and used to study the stability properties. Now, this same admittance $Y_{M S}$ is used to study the transparency properties, because it directly describes what the human operator feels.

Transparency is a performance measure indicating how well the human operator perceives the remote environment. Ideally, the teleoperation system should behave as a massless, infinitely stiff connection between the human operator and the environment Raju et al. 1988. In that case, the operator feels as if he/she is directly interacting with the remote environment. Lawrence [1993 defined transparency as follows: for a completely transparent system the impedance transmitted to or felt by the operator has to be equal to the impedance of the remote environment. As the impedance felt by the operator is the inverse of the admittance $Y_{M S}$, this definition becomes:

$$
Y_{M S, \text { desired }}=\frac{1}{Z_{e}}
$$

The Position-Force controller as defined in section 2 supposes a low-impedance type master with pure open-loop force control. As a consequence, the dynamics of the master are not cancelled and the best achievable transparency is:

$$
Y_{M S\left(Z_{e}\right)}(s)=\frac{1}{M_{m} s+B_{m}+\mu \lambda Z_{e}}
$$

This means that the operator always feels the full dynamics of the master, including the friction of the master. These dynamics have been called the tool dynamics by Salcudean et al. 1999]. However, as low-impedance-type haptic devices like the PHANToM from Sensable Inc. are characterized by both low inertia and low friction/damping values, this does not necessarily significantly deteriorate the overall transparency ${ }^{7}$. As a surgeon depends largely on the perception and interpretation of the stiffness of tissues and structures De Gersem et al., 2005], special attention is paid here to stiffness transparency Christiansson and van der Helm, 2007, Willaert et al., 2010b. A good measure for stiffness transparency is the stiffness the human operator feels when manipulating the master quasi-statically for an environment that behaves as a pure spring $\left(Z_{e}=K_{e}\right)$. Assuming a rigid slave, this measure can be deduced from the admittances defined in section 5, which gives for both the P-F scheme and the PD-F scheme 8

$$
\lim _{s \rightarrow 0} Y_{M S\left(K_{e}\right)}(s)=\frac{K_{p}+K_{e}}{\mu \lambda\left(K_{p} K_{e}\right)}
$$

The operator feels a stiffness that is proportional to $\mu \lambda$ and to the series connection of $K_{p}$ and $K_{e}$. For a flexible slave, assuming the model given in subsection 6.2, the stiffness the human operator feels when manipulating the master

\footnotetext{
${ }^{7}$ Humans are used to manipulate the environment through physical tools with a non-zero mass/inertia (e.g. a hammer, a screwdriver, ...).

${ }^{8}$ The velocity feedforward term of the PD-F scheme does not change this measure for the stiffness transparency as it is a static property.
} 
quasi-statically is for both the $\mathbf{P}-\mathbf{F}$ scheme and the PD-F scheme:

$$
\lim _{s \rightarrow 0} Y_{M S\left(K_{e}\right)}(s)=\frac{K_{p} K_{e}+K_{e} K_{s}+K_{s} K_{p}}{\mu \lambda\left(K_{s} K_{p} K_{e}\right)}
$$

The operator now feels a stiffness that is proportional to $\mu \lambda$ and to the series connection of $K_{e}, K_{p}$ and $K_{s}$, with $K_{s}$ the structural stiffness of the slave. Thus, for a correct reflection of the environment stiffness, $\mu \lambda$ should be as close as possible to 1 and both the gain of the position controller of the slave $K_{p}$ and the structural stiffness of the slave $K_{s}$ should be as large as possible. The requirement on $\mu \lambda$ is an example of the well known stability-transparency trade-off. The other two requirements however, show that there is not always a trade-off between stability and transparency. In section 5 and 6 it is shown that larger values of both $K_{p}$ and $K_{s}$ also improve the passivity of $Y_{M S}$.

\section{Experimental results}

In this section, the correctness of the derived conditions for passivity of the admittance $Y_{M S}$ is systematically verified through experiments with a conscious choice of different environments and operators. The experiments are done on the 1-d.o.f. experimental master-slave setup described in section 2 and shown in Fig 13 . The parameters for both control schemes are those of Table1. Recall that for these parameters the teleoperation system is not absolutely stable for the $\mathbf{P}-\mathbf{F}$ scheme nor for the $\mathbf{P D}-\mathbf{F}$ scheme.

For four different environments, i.e. three springs and one hard contact, the passivity of the admittance $Y_{M S}$ is calculated and verified experimentally. These environments are assumed to be pure stiffnesses in order to calculate the passivity of the admittance $Y_{M S\left(K_{e}\right)}$. The numerical values of these stiffnesses are: $K_{e}=100,300,880$ and $\pm 27000 \mathrm{~N} / \mathrm{m}$. Fig. 15 shows the Bode plots of $Y_{M S\left(K_{e}\right)}$ for these four values of $K_{e}$ for the P-F scheme and the PD-F scheme.

For each of these different environments, stable interaction with a human operator is not a sufficient demonstration of the passivity of $Y_{M S\left(K_{e}\right)}$, since a human

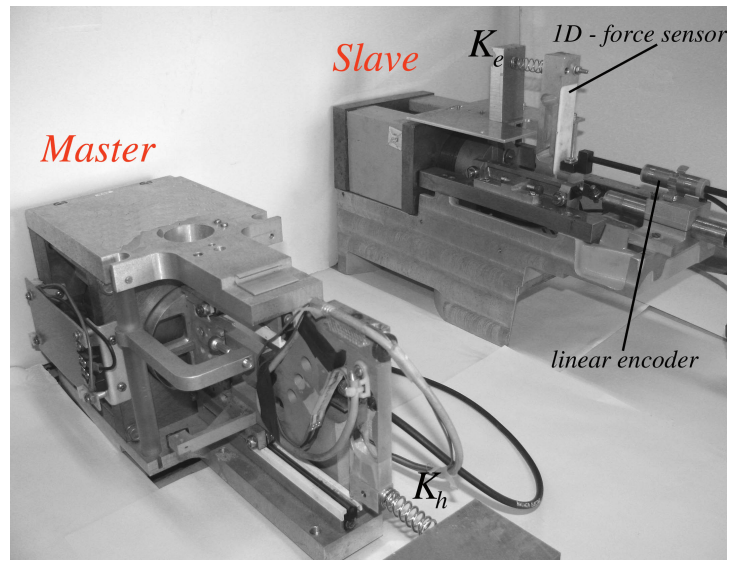

Figure 13: The 1 d.o.f experimental teleoperation setup in the case that both the environment and the operator are a pure stiffness $\left(K_{e}\right.$ and $\left.K_{h}\right)$. 


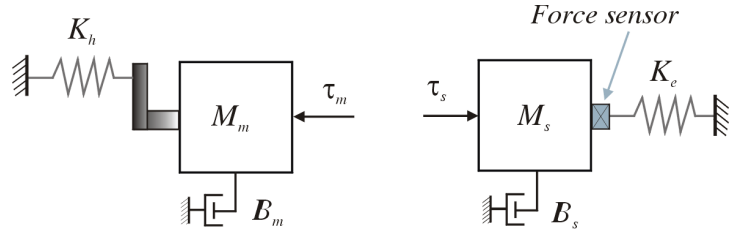

Figure 14: A schematic illustration of the experimental master-slave system with springs as both environment and operator.

Table 2: Experimental results, no low-pass filter in the loop. For four different springlike environments, the passivity of $Y_{M S\left(K_{e}\right)}$ is given and the stability of the interaction with three different operators is described, i.e. a soft spring $\left(K_{h}=250 \mathrm{~N} / \mathrm{m}\right)$, a human operator and a hard object $\left(K_{h}= \pm 50000 \mathrm{~N} / \mathrm{m}\right)$.

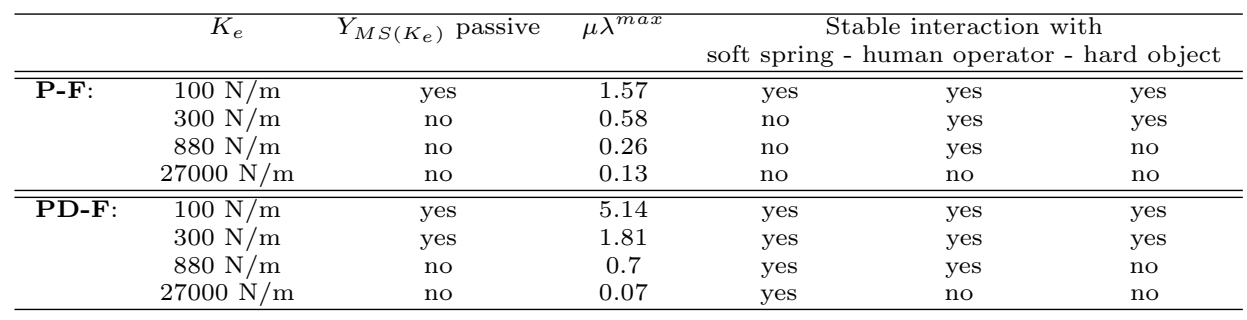

operator tends to stabilize the interaction. To experimentally verify the passivity of $Y_{M S\left(K_{e}\right)}$, the operator should behave as a worst case passive operator. Therefore, the stability of the interaction between the teleoperation system and an operator is checked for three different types of operators: a human operator, a soft spring and a hard object. Fig 13 and Fig 14 show a picture and a schematic illustration of the master-slave system with a spring as environment and as operator.

Table 2 summarizes the calculation and the experimental findings in case that no low-pass filter is used, while Table 3 does so for the case of a $5 \mathrm{~Hz}$ low-pass filter present in the loop (see 6.1). Both tables show, for each environment, whether $Y_{M S\left(K_{e}\right)}$ is passive for the parameters of Table 1. i.e. with $\mu=\lambda=1$, what $\mu \lambda^{\max }$ is and how each environment/operator combination behaves.

For the soft spring and the hard object as operator, the system was considered stable, if a small, manually given, force input at the master did not result in growing oscillations. As could be expected, none of the 'operators' causes an

Table 3: Experimental results, $5 \mathrm{~Hz}$ low-pass filter in the loop

\begin{tabular}{ccccccc}
\hline & $K_{e}$ & $Y_{M S\left(K_{e}\right)}$ passive & $\mu \lambda^{\text {max }}$ & \multicolumn{2}{c}{$\begin{array}{c}\text { Stable interaction with } \\
\text { soft spring - human operator - hard object }\end{array}$} \\
\hline \hline PD-F: & $100 \mathrm{~N} / \mathrm{m}$ & yes & 1.02 & yes & yes & yes \\
& $300 \mathrm{~N} / \mathrm{m}$ & no & 0.36 & no & yes & yes \\
& $880 \mathrm{~N} / \mathrm{m}$ & no & 0.15 & no & no & no \\
$27000 \mathrm{~N} / \mathrm{m}$ & no & 0.07 & no & no & no \\
\hline
\end{tabular}




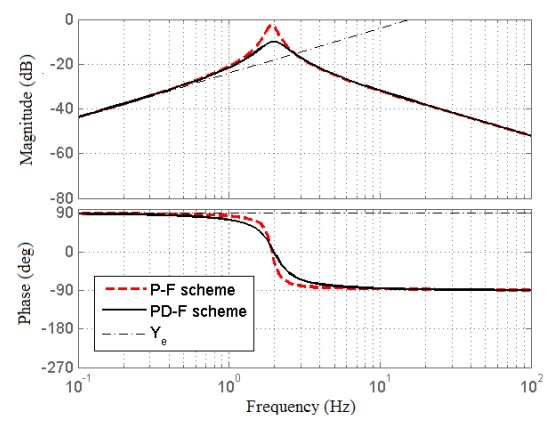

(a) $K_{e}=100 \mathrm{~N} / \mathrm{m}$

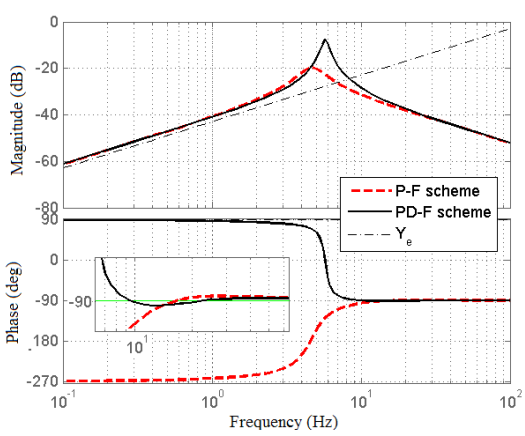

(c) $K_{e}=880 \mathrm{~N} / \mathrm{m}$

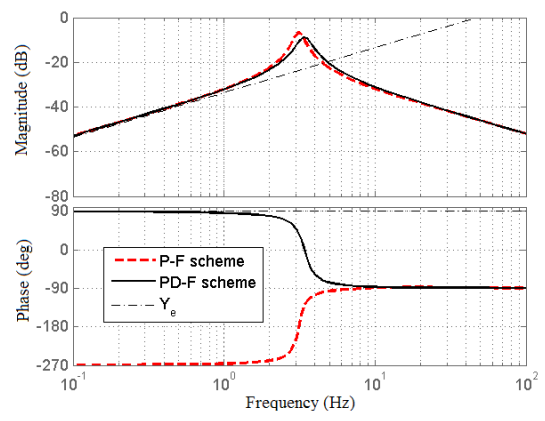

(b) $K_{e}=300 \mathrm{~N} / \mathrm{m}$

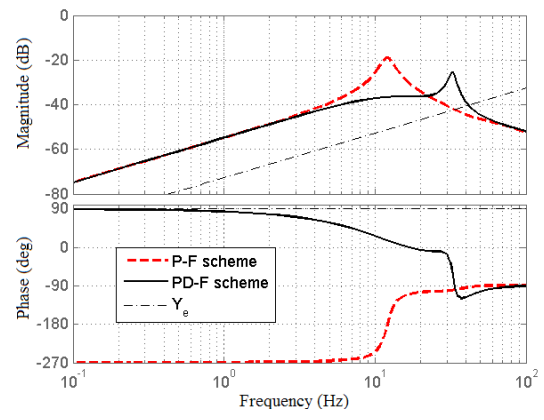

(d) $K_{e}=27000 \mathrm{~N} / \mathrm{m}$

Figure 15: The bode plot of $Y_{e}$ and $Y_{M S\left(K_{e}\right)}$ for the P-F scheme and the PD-F scheme for four different values of $K_{e}$. These plots provide both a check of the passivity of $Y_{M S}$ and a check of the transparency properties.

instability when the admittance $Y_{M S\left(K_{e}\right)}$ is passive. For an active admittance $Y_{M S\left(K_{e}\right)}$, the stability behaviour with the soft spring and the hard object as operator is in correspondence with a calculation of stability of the transfer function $T_{c l}$ :

$$
T_{c l}=\frac{1}{1+Z_{h} Y_{M S\left(K_{e}\right)}},
$$

representing the closed-loop interaction of the operator and the admittance $Y_{M S\left(K_{e}\right)}$. Both the soft spring and the hard object are assumed to be pure stiffnesses in order to calculate the stability of this closed-loop. The numerical values of these stiffnesses are: $K_{h}=250$ and $\pm 50000 \mathrm{~N} / \mathrm{m}$. Note that for closed-loop stability, the poles of the closed-loop transfer function $T_{c l}$ must have a negative real part.

For a human as operator, the stability behaviour is less straightforward. As mentioned in section 5, for the parameters of Table 1, the maximum environment stiffness $K_{e}^{\max }$ for which $Y_{M S\left(K_{e}\right)}$ is passive is $161 \mathrm{~N} / \mathrm{m}$ for the P-F scheme and $580 \mathrm{~N} / \mathrm{m}$ for the PD-F scheme. However, frequent testing ${ }^{9}$ shows

\footnotetext{
${ }^{9}$ Experimental testing shows that, for human operators, it can be hard to say whether an interaction is stable or not. Typically there is a range of stiffnesses for which the interaction is no longer perceived as really stable neither yet unstable.
} 
that a human operator is able to interact stably with stiffnesses that are a factor 5 to 10 larger than these bounds defined by passivity of $Y_{M S\left(K_{e}\right)}$. This factor depends among others on the posture and the grip of the human operator. This finding corresponds to the well-known idea that guaranteeing stability for any passive operator can be conservative. The behaviour of the human operator and the coupling with the admittance $Y_{M S}$ will be addressed in future research.

The main conclusion from these experiments is the very good correspondence between the derived passivity and stability conditions and the experimental behaviour. For the experiments where the human operator is replaced by either a soft spring or a hard object, all experimental findings are in accordance with both the passivity and the closed-loop stability calculations. For the experiments with a human operator, the interaction was always stable when the system was calculated to be passive. Furthermore, the experiments clearly demonstrate the negative effect of a low-pass filter in the loop.

\section{Conclusions and future work}

This paper describes a task-based mechatronic analysis based on a new method called Bounded Environment Passivity. This method studies the passivity of the one-port network comprising the teleoperation system and the environment, represented by the admittance $Y_{M S}$. This is the admittance with which the human operator interacts. The passivity of this one-port network is studied separately for a pure mass environment and a pure stiffness environment, which facilitates analytic analyses.

In the paper, the method is applied to the classical Position-Force controller. Depending on the complexity of the hardware-model and/or controller, analytic or numerical analysis of Bounded Environment Passivity results in conditions on both hardware and control parameters. These conditions, together with some transparency considerations, show the importance of the dynamic properties of both the master and the slave for reliable haptic feedback. Concerning the design of the slave, some rules of thumb are clear. First, the slave should be as lightweight as possible. Second, the slave should be as stiff as possible. Although these requirements are the design criteria for a lot of applications, the importance of these dynamic properties is often not explicitly discussed in the field of teleoperation. In some sense, these two rules of thumb are conflicting. Therefore, in future research, the optimization of these dynamic properties will be addressed.

In order to achieve good stiffness transparency, the value of $\mu \lambda$ should be 1 . For which range of environment stiffnesses the passivity condition allows $\mu \lambda=1$, depends on different parameters of the system. The conditions presented in this paper, however, show that even for a very well designed multi-d.o.f. system for minimally invasive surgery, the dynamic properties of the system are unlikely to allow a large range of environment stiffnesses for which the value of $\mu \lambda$ can be 1. Therefore, in ongoing research, it is being investigated which extensions of the Position-Force controller result in better Bounded Environment Passivity properties Willaert et al. 2010a.

The experiments described in this paper show that by using different kinds of op- 
erators, i.e. a soft spring, a hard object and a human operator, one can reliably test the passivity of the admittance $Y_{M S}$. The experiments also showed that guaranteeing stable interaction with all possible passive operators can result in too conservative results. Therefore, in future work, it should be investigated how also bounds on the operator can be included in the analysis, e.g. by placing a shunt impedance in front of the admittance $Y_{M S}$.

Strictly speaking, the presented results are only conclusive in the case of a pure mass and a pure spring as environment. Next to this, it is assumed that the results can be generalized to mass-spring-damper systems as environment. This is illustrated in Appendix $\mathrm{D}$ for the Position-Force controller, but a rigorous proof for this assumption remains a topic for further research.

A last important finding from this paper is that it is hard to come up with general conclusions about 'the' Position-Force controller. The best example for this is the fact that the PD-F scheme shows significantly better Bounded Environment Passivity properties than the $\mathbf{P}-\mathbf{F}$ scheme, although the the $\mathbf{P}-\mathbf{F}$ scheme can be absolutely stable while the PD-F scheme cannot. This demonstrates that a small change in the controller definition can significantly affect the passivity properties. Such a finding demonstrates the need for careful stability analyses, preferably resulting in analytic conditions.

\section{ACKNOWLEDGMENTS}

This work was supported by a PhD grant from the Institute for the Promotion of Innovation through Science and Technology in Flanders (I.W.T.-Vlaanderen), by two I.W.T projects (IWT/OZM/080086 and IWT/OZM/080003), by the K.U.Leuven BOF-IDO/05/008 project and by an FP7-People Marie Curie Reintegration Grant, PIRG03-2008-231045.

\section{A Appendix A: Mathematical methodology}

This appendix shows the methodology that is often used throughout the paper for deriving analytic expressions:

- Conditions that are of the following form:

$$
a \omega^{4}+b \omega^{2}+c \geq 0, \forall \omega
$$

can be written as:

$$
a x^{2}+b x+c \geq 0, \forall x \geq 0
$$

This holds $\Leftrightarrow a \geq 0$ and $c \geq 0$ and ( $b \geq 0$ or $\left.b^{2}-4 a c \leq 0\right)$.

- Checking the positive realness of a general transfer function of the form $\frac{A+j B}{C+j D}$ is done as follow:

$$
\Re\left[\frac{A+j B}{C+j D}\right]=\frac{A \cdot C+B \cdot D}{C^{2}+D^{2}}
$$

As $C^{2}+D^{2}$ if always positive, $\frac{A+j B}{C+j D}$ is positive real $\Leftrightarrow A . C+B . D \geq 0$ 


\section{B Appendix B: Bounded Environment Passivity for a pure mass environment}

This appendix proves that a Position-Force controller as defined in section 2 experiences no stability problems when interacting with a pure mass as environment. To do so, the Bounded Environment Passivity analysis of section 5 is repeated here for a pure mass $M_{e}$ instead of a pure spring $K_{e}$ as environment. For the P-F scheme, the admittance $Y_{M S\left(M_{e}\right)}$ can now be written as:

$$
Y_{M S\left(M_{e}\right)}=\frac{\left(M_{e}+M_{s}\right) s^{2}+\left(K_{v}+B_{s}\right) s+K_{p}}{\left(M_{m} s+B_{m}\right)\left(\left(M_{e}+M_{s}\right) s^{2}+\left(K_{v}+B_{s}\right) s+K_{p}\right)+\mu \lambda K_{p} M_{e} s}
$$

The requirement $\Re\left(Y_{M S\left(M_{e}\right)}(j \omega)\right) \geq 0$ can be expressed as:

$$
\begin{array}{r}
\left(M_{e}^{2} B_{m}+2 M_{e} B_{m} M_{s}+M_{s}^{2} B_{m}\right) \omega^{4}+\left(-2 K_{p} B_{m} M_{e}-2 K_{p} B_{m} M_{s}+B_{m} K_{v}^{2}+2 K_{v} B_{m} B_{s}+\right. \\
\left.B_{s} K_{p} \mu \lambda M_{e}+K_{v} K_{p} \lambda M_{e}+B_{m} B_{s}^{2}\right) \omega^{2}+B_{m} K_{p}^{2} \geq 0, \forall \omega \geq 0,
\end{array}
$$

This is of the form $a x^{2}+b x+c \geq 0, \forall x \geq 0$ with $x=\omega^{2}$. It is trivial to show that $(a \geq 0)$ and $(c \geq 0)$ hold. The condition $\left(b \geq 0\right.$ or $\left.b^{2}-4 a c \leq 0\right)$ results in the following conditions on $\mu \lambda$ :

$$
\begin{gathered}
\mu \lambda \geq-\frac{B_{m}\left(B_{s}+K_{v}\right)}{K_{p} M_{e}}+2 \frac{\left(M_{e}+M_{s}\right) B_{m}}{\left(K_{v}+B_{s}\right) M_{e}} \\
-\frac{B_{m}\left(B_{s}+K_{v}\right)}{K_{p} M_{e}} \leq \mu \lambda \leq-\frac{B_{m}\left(B_{s}+K_{v}\right)}{K_{p} M_{e}}+4 \frac{\left(M_{e}+M_{s}\right) B_{m}}{\left(K_{v}+B_{s}\right) M_{e}}
\end{gathered}
$$

As the lower boundary of condition (58) is always negative and the lower boundary of condition (57) always falls in between the upper and lower boundary of condition (58), $\left(b \geq 0\right.$ or $\left.b^{2}-4 a c \leq 0\right)$ always holds for positive $\mu \lambda$ values. This proofs that for the P-F scheme, the admittance $Y_{M S\left(M_{e}\right)}$ is always passive independent of the value of $M_{e}$. As for the PD-F scheme a similar derivation can be made, the above derivation allows the formulation of the following theorem:

THEOREM 4 (Passive Position-Force Controller for $Z_{e}=M_{e} . s$ )

The combination of the teleoperation system (3) and (4) with a Position-Force controller defined by (6) and (7) or by (6) and (8) and a pure mass environment, results in a one-port network $Y_{M S\left(M_{e}\right)}$ that is passive for all positive parameters of the system, independent of the value of $M_{e}$.

\section{Appendix C: Bounded Environment Passivity conditions for the PD-F scheme}

This appendix shows the full analytic conditions resulting from the requirement that $\Re\left(Y_{M S\left(K_{e}\right)}\right) \geq 0$ for the PD-F scheme: [ $[59)$ or $\left.(60)\right]$ and $[\sqrt{61]}$ or $[(62)$ and $[63]]]$ :

$$
K_{e}^{\max } \leq \frac{B_{s} K_{p}}{B_{s}+2 K_{v}} \Rightarrow \mu \lambda \leq \frac{B_{m}\left(K_{e}^{\max }+K_{p}\right)^{2}}{\left(B_{s} K_{p}-K_{v} K_{e}^{\max }\right) K_{e}^{\max }}
$$




$$
\begin{gathered}
K_{e}^{\max }>\frac{B_{s} K_{p}}{B_{s}+2 K_{v}} \Rightarrow \mu \lambda \leq \frac{4 B_{m}\left(B_{s}+K_{v}\right)}{B_{s}} \\
\mu \lambda \leq \frac{B_{m}\left(B_{s}+K_{v}\right)^{2}-2\left(K_{p}+K_{e}\right) M_{s} B_{m}}{M_{s} K_{v} K_{e}} \\
\mu \lambda \leq \frac{B_{m}\left(B_{s}+K_{v}\right)}{M_{s} K_{e} K_{v}^{2}}\left(\left(B_{s}+K_{v}\right) K_{v}-2 M_{s} K_{p}+2 \sqrt{M_{s}^{2} K_{p}^{2}+M_{s} K_{v}^{2} K_{e}-K_{p} K_{v} B_{s} M_{s}}\right) \\
\mu \lambda \geq \frac{B_{m}\left(B_{s}+K_{v}\right)}{M_{s} K_{e} K_{v}^{2}}\left(\left(B_{s}+K_{v}\right) K_{v}-2 M_{s} K_{p}-2 \sqrt{M_{s}^{2} K_{p}^{2}+M_{s} K_{v}^{2} K_{e}-K_{p} K_{v} B_{s} M_{s}}\right)
\end{gathered}
$$

\section{Appendix D: Extension to mass-spring-damper systems as environment}

In section 5 the following assumption is described:

$$
\begin{gathered}
\begin{cases}\Re\left(Y_{M S\left(M_{e}\right)}\right) \geq 0 & \text { with } M_{e} \in\left[0, M_{e}^{\text {max }}\right] \\
\Re\left(Y_{M S\left(K_{e}\right)}\right) \geq 0 & \text { with } K_{e} \in\left[0, K_{e}^{\text {max }}\right]\end{cases} \\
\Downarrow \\
\Re\left(Y_{M S\left(Z_{e}\right)}\right) \geq 0, \forall Z_{e}=M_{e} s+B_{e}+\frac{K_{e}}{s} \text { with }\left\{\begin{array}{l}
M_{e} \in\left[0, M_{e}^{\text {max }}\right] \\
B_{e} \in[0, \infty] \\
K_{e} \in\left[0, K_{e}^{\text {max }}\right]
\end{array}\right.
\end{gathered}
$$

Currently, there is no proof for this general assumption, nor a counterexample. We tested this assumption for the P-F scheme, the PD-F scheme and also for the PD-F-F ${ }_{h}$ scheme (this is a three-channel controller described by Willaert et al. 2010a ) and for all three control schemes the assumption was confirmed. Hereunder, this assumption is illustrated for the PD-F scheme.

For the PD-F scheme and an environment $Z_{e}=M_{e} s+B_{e}+\frac{K_{e}}{s}$, the admittance $Y_{M S\left(Z_{e}\right)}$ can be written as:

$$
Y_{M S}=\frac{\left(M_{e}+M_{s}\right) s^{2}+\left(K_{v}+B_{s}+B_{e}\right) s+\left(K_{p}+K_{e}\right)}{\left(M_{m} s+B_{m}\right)\left(\left(M_{e}+M_{s}\right) s^{2}+\left(K_{v}+B_{s}+B_{e}\right) s+\left(K_{p}+K_{e}\right)\right)+\mu \lambda\left(K_{v} s+K_{p}\right)\left(M_{e} s+B_{e}+\frac{K_{e}}{s}\right)} .
$$

For this admittance, the positive real part condition, i.e. $\Re\left(Y_{M S}\right) \geq 0$ for all $\omega$, can be expressed as a $2^{\text {nd }}$ order inequality in $x=\omega^{2}$. Consequently, the resulting conditions have been calculated analytically. However, compared to the conditions obtained in section 5 these conditions are very cumbersome and are therefore not put in this appendix. In Fig. 16. the most restricting analytic condition is shown for a range of values of $M_{e}$ and some values of $B_{e}$, for the parameters of Table 1 . This figure illustrates the above mentioned assumption and shows that for the PD-F scheme both the environment mass and the environment damping relax the condition on the environment stiffness. The fact that damping in the environment relaxes the condition on the environment stiffness is no surprise. If one can assume some minimal damping in the environment, it is possible to come up with less conservative conditions. However, as already mentioned here and in the introduction, every extra parameter in the admittance $Y_{M S}$ can make the resulting conditions more cumbersome. By restricting the analysis to a pure mass environment and a pure stiffness environment separately, the resulting conditions remain manageable. 


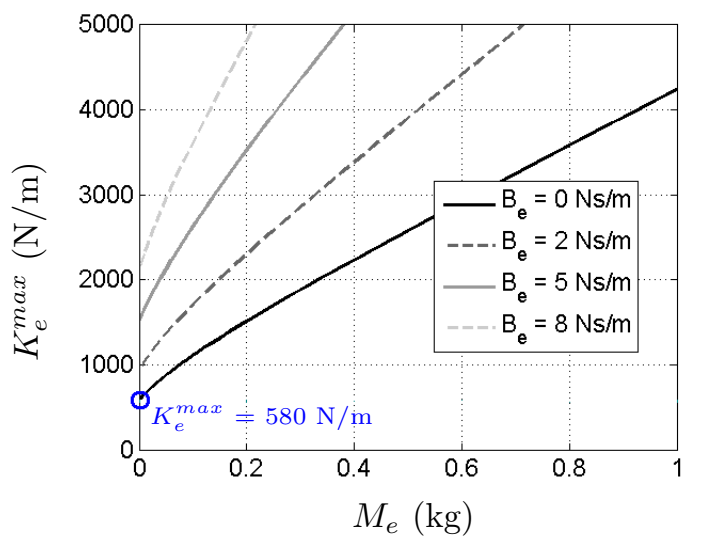

Figure 16: The maximum value for $K_{e}$ in function of $M_{e}$ and $B_{e}$ for which $Y_{M S\left(Z_{e}\right)}$ is positive real, i.e. passive. For $M_{e}=B_{e}=0$, the maximum environment stiffness is $580 \mathrm{~N} / \mathrm{m}$, which corresponds to the value found in section 5 .

\section{References}

R. Adams and B. Hannaford. Stable haptic interaction with virtual environments. IEEE transactions on robotics and automation, 15(3):465-474, June 1999.

R. Adams and B. Hannaford. Control law design for haptic interfaces to virtual reality. IEEE Transactions on control systems technology, 10(1):3-13, January 2002.

I. Aliaga, A. Rubio, and E. Sanchez. Experimental quantitative comparison of different control architectures for master-slave teleoperation. IEEE Transactions on Control Systems Technology, 12(1):2-11, January 2004.

R. Anderson and M. Spong. Bilateral control of teleoperators with time delay. In Proceedings of IEEE international conference on Systems, Man \& Cybernetics, volume 1, pages 131-138, Bejing, China, Aug 1988.

I. Bankman, K. Gruhen, H. Halperin, A. Popel, A. Guerci, and J. Tsitlik. Identification of dynamic mechanical properties of the human chest during manual cardiopulmonary resuscitation. IEEE Transactions on Biomedical Engineering, 37(2):211-217, February 1990.

Y. Bu, R. Daniel, and P. McAree. Stability analysis of force reflecting telerobotic systems. In Proceedings of IEEE International Conference on Intelligent Robots and Systems, volume 3, pages 1374-1379, Osaka, Japan, November 1996.

M. Cavusoglu, A. Sherman, and F. Tendick. Bilateral controller design for telemanipulation in soft environments. IEEE Transactions on Robotics and Automation, 18(4):641-647, August 2002. 
H. Cho and J. Park. Impedance control with variable damping for bilateral teleoperation under time delay. JSME International Journal Series C, 48(4): 695-703, 2005.

G. Christiansson and F. van der Helm. The low-stiffness teleoperator slave - a trade-off between stability and performance. Int. Journal of Robotics Research, 26(3):287-299, March 2007.

J. Colgate and N. Hogan. Robust control of dynamically interacting systems. International Journal on Control, pages 65-88, 1988.

J. E. Colgate. Robust impedance shaping telemanipulation. IEEE Transactions on robotics and automation, 9(4):374-384, August 1993.

R. Daniel and P. McAree. Fundamental limits of performance for force reflecting teleoperation. The International Journal of Robotics Research, 17(8):811-830, August 1998.

G. De Gersem, H. Van Brussel, and F. Tendick. Reliable and enhanced stiffness perception in soft-tissue telemanipulation. The international Journal of Robotics Research, 24(10):805-822, October 2005.

B. Deml, T. Ortmaier, and U. Seibold. The touch and feel in minimally invasive surgery. In Proceedings of International Workshop on Haptic Audio Visual Environments and their Applications, pages 33-38, Ontario, Canada, October 2005.

C. Desoer and M. Vidyasagar. Feedback systems: Input-output properties. Academic Press (New York), 1975.

S. K. Dwivedy and P. Eberhard. Dynamic analysis of flexible manipulators, a literature review. Mechanism and Machine Theory, 41:749777, 2006.

K. Fite, L. Shao, and M. Goldfarb. Loop shaping for transparency and stability robustness in bilateral telemanipulation. IEEE Transactions on Robotics and Automation, 20(3):620-624, June 2004.

K. Fite, J. Speich, and M. Goldfarb. Transparency and stability robustness in two-channel bilateral telemanipulation. Journal of Dynamic Systems, Measurement, and Control, 123:400-407, 2001.

A. Haddadi and K. Hashtrudi-Zaad. A new robust stability analysis and design tool for bilateral teleoperation control systems. In Proceedings of the IEEE International Conference on Robotics and Automation, pages 663-670, Pasadena, CA, USA, May 2008.

B. Hannaford. A design framework for teleoperators with kinesthetic feedback. IEEE Transactions on Robotics and Automation, 5(4):426-434, August 1989a.

B. Hannaford. Stability and performance trade-offs in bilateral telemanipulation. In Proceedings of the IEEE International Conference on Robotics and Automation, pages 1764-1767, 1989b. 
B. Hannaford and R. Anderson. Experimental and simulation studies of hard contact in force reflecting teleoperation. In Proceedings of the IEEE International Conference on Robotics and Automation, Phil., PA, 1988.

K. Hashtrudi-Zaad and S. Salcudean. Analysis of control architectures for teleoperation systems with impedance/admittance master and slave manipulators. The international Journal of Robotics Research, 20(6):419-445, June 2001.

S. Haykin. Active network theory. Addison-Wesley, 1970.

N. Hockstein, C. Gourin, R. Faust, and D. Terris. A history of robots: from science fiction to surgical robotics. Journal of Robotic Surgery, 1:113-118, 2007.

N. Hogan. Controllling impedance at the man/machine interface. In Proceedings of the IEEE International Conference on Robotics and Automation, pages 1626-1631, Scottsdale, AZ, 1989.

C. Lawn and B. Hannaford. Performance testing of passive communication and control in teleoperation with time delay. In Proceedings of the IEEE International Conference on Robotics and Automation, pages 776-783, Atlanta, GA, May 1993.

D. Lawrence. Stability and transparency in bilateral teleoperation. IEEE transactions on robotics and automation, 9(5):624-637, October 1993.

F. Llewellyn. Some fundamental properties of transmission systems. In Proc. IRE, volume 40, pages 271-283, 1952.

S. Misra and A. Okamura. Environment parameter estimation during bilateral telemanipulation. In Proceedings of Symposium on Haptic Interfaces for Virtual Environment and Teleoperator Systems, pages 301-307, Virginia, USA, March 2006.

A. Okamura. Methods for haptic feedback in teleoperated robot-assisted surgery. Industrial Robot: An international Journal, 31(6):499-508, 2004.

G. Raisbeck. A definition of passive linear networks in terms of time and energy. Journal of Applied Physics, 12:1510-1514, 1954.

G. R. Raju, G. Verghese, and T. Sheridan. Design issues in 2-port network models of bilateral remotemanipulation. In Proceedings of the IEEE International Conference on Robotics and Automation, volume 3, pages 1316-1321, Scottsdale, AZ, May 1988.

J. Rosen, D. Jeffrey, S. De, M. Sinanan, and B. Hannaford. Biomechanical properties of abdominal organs in vivo and postmortem under compression loads. Journal of Biomechanical Engineering, 130(2):1-17, April 2008.

S. Salcudean, K. Hashtrudi-Zaad, S. Tafazoli, S. P. Dimaio, and C. Reboulet. Bilateral matched-impedance teleoperation with application to excavator control. IEEE Control Systems Magazine, 6:29-37, 1999. 
H. I. Son and D. Y. Lee. Enhancement of kinesthetic perception for microsurgical teleoperation using impedance-shaping. In Proceedings of the 30th Annual Int. IEEE EMBS Conference, pages 1939-1942, Vancouver, B.C., Canada, August 2008.

M. Tavakoli, A. Aziminejad, R. Patel, and M. Moallem. Enhanced transparency in haptics-based master-slave systems. In Proceedings of the 2007 American Control Conference, pages 1455-1460, New York City, USA, July 2007.

M. Tavakoli and R. Howe. Haptic effect of surgical teleoperator flexibility. Int. Journal of Robotics Research, 28(10):1289-1302, October 2009.

G. Tholey, J. Desai, and A. Castellanos. Force feedback plays a significant role in minimally invasive surgery. Annals of surgery, 241(1):102-109, January 2005 .

E. Vander Poorten. Improving Haptic Fidelity for Intuitive Manipulation in Scaled Teleoperation and Virtual Environment. PhD thesis, University of Kyoto, 2007.

E. Vander Poorten, Y. Yokokohji, and Y. Tsuneo. Stability analysis and robust control for fixed-scale teleoperation. Advanced Robotics, 20:681-706, 2006.

C. R. Wagner, N. Stylopoulos, and R. D. Howe. The role of force feedback in surgery: Analysis of blunt dissection. In The 10th Symp. on Haptic interfaces for virtual environment and teleoperator systems, Orlando, 2002.

J. Walraevens, B. Willaert, G. De Win, A. Ranftl, J. De Schutter, and J. Vander Sloten. Correlation between compression, tensile and tearing tests on healthy and calcified aortic tissues. Medical Engineering \& Physics, 30:1098 - 1104, 2008 .

B. Willaert, B. Corteville, D. Reynaerts, H. V. Brussel, and E. V. Poorten. Bounded environment passivity of the classical position-force teleoperation controller. In Proceedings of the IEEE/RSJ Int. Conference on Intelligent Robots and Systems, pages 4622-4628, St. Louis, MO, USA, October 2009.

B. Willaert, B. Corteville, D. Reynaerts, H. V. Brussel, and E. V. Poorten. Transparency trade-offs for a 3-channel controller revealed by the bounded environment passivity method. In The third Int. Conf. on Advances in Computer-Human Interactions (ACHI), pages 66-72, St. Maarten, February 2010a.

B. Willaert, P. Goethals, D. Reynaerts, H. V. Brussel, and E. V. Poorten. Advances in Haptics, chapter 13: Transparent and Shaped Stiffness Reflection for Telesurgery, pages 259-282. IN-TECH, 2010b.

Y. Yokokohji and T. Yoshikawa. Bilateral control of master-slave manipulators for ideal kinesthetic coupling. IEEE transactions on robotics and automation, 10(5):605-620, October 1994. 